\title{
Diffusion coefficients of alloying elements in dilute Mg alloys: A
}

\section{comprehensive first-principles study}

\author{
Bi-Cheng Zhou*, Shun-Li Shang, Yi Wang, Zi-Kui Liu \\ Department of Materials Science and Engineering, The Pennsylvania State University, \\ University Park, PA 16802, USA
}

\begin{abstract}
First-principles calculations based on density functional theory have been used to calculate the temperature-dependent dilute tracer diffusion coefficients for 47 substitutional alloying elements in hexagonal closed packed (hcp) $\mathrm{Mg}$ by combining transition state theory and an 8-frequency model. The minimum energy pathways and the saddle point configurations during solute migration are calculated with the climbing image nudged elastic band method. Vibrational properties are obtained using the quasi-harmonic Debye model with inputs from first-principles calculations. An improved generalized gradient approximation of PBEsol is used in the present first-principles calculations, which is able to well describe both vacancy formation energies and vibrational properties. It is found that the solute diffusion coefficients in dilute hcp $\mathrm{Mg}$ are roughly inversely proportional to bulk modulus, which reflects the solutes' bonding to $\mathrm{Mg}$. Transition metal elements with $d$ electrons show strong interactions with $\mathrm{Mg}$ and have large diffusion activation energies. Correlation effects are not negligible for solutes $\mathrm{Ca}, \mathrm{Na}, \mathrm{Sr}, \mathrm{Se}, \mathrm{Te}$, and $\mathrm{Y}$, in which the direct solute migration barriers are much smaller than the solvent $(\mathrm{Mg})$ migration barriers. Calculated diffusion coefficients are in remarkable agreement with available experimental data in the literature.

* Corresponding author. Tel.: +1 8144416349.

E-mail address: zhoubicheng@gmail.com, bicheng.zhou@psu.edu (B.C. Zhou).
\end{abstract}


Keywords: Magnesium alloys; Bulk diffusion; First-principles calculation; Kinetics

\section{Introduction}

In recent years, magnesium $(\mathrm{Mg})$ alloys have received an increasing interest due to their low density, earth abundance, high specific strength, and good castability [1]. These properties make $\mathrm{Mg}$ alloys attractive for automotive, aerospace, and other light-weight structural applications [2]. The majority of $\mathrm{Mg}$ alloys derives their mechanical properties from precipitation hardening [3], while the study of precipitation process demands accurate thermodynamic and kinetic (diffusion) data. Thermodynamics of Mg alloys has been extensively studied, and several comprehensive thermodynamic databases have been established [4]. However, the kinetics of Mg alloys has been studied to a far lesser extent, especially diffusion coefficients of various solutes in $\mathrm{Mg}$. Due to the issues related to corrosion, oxidation, and contamination during sample preparation in diffusion measurements, few experimental data are available in the literature for diffusion coefficients of solutes in $\mathrm{Mg}$ [5].

For the investigation of kinetic processes in Mg alloys in the solid state, such as creep [6], solute strengthening [7, 8], solution treatment and aging [9], reliable diffusion data and detailed insights into diffusion of solutes in $\mathrm{Mg}$ are desperately needed. For example, the knowledge of diffusion coefficients can help to determine the desirable aging time to achieve peak hardness in precipitation-hardened $\mathrm{Mg}$ alloys [3]. Wrought $\mathrm{Mg}$ alloys have seen very little implementation in the automotive industry because of their poor formability at room temperature [6]. To improve the formability of wrought $\mathrm{Mg}$ alloys, proper alloying additions can be selected by evaluating their solute drag propensity at the grain boundaries [10] to mitigate the basal plane texture 
formation due to the inhomogeneous deformation of hcp $\mathrm{Mg}$. This propensity greatly depends on their diffusion coefficients based on Cahn's solute drag theory [11]. Diffusion of solutes around the dislocation core structure in $\mathrm{Mg}$ also plays an important role in understanding the origin of many plastic phenomena such as dynamic strain aging [7] and plastic instabilities [12]. Therefore, the information of solute diffusion coefficients in $\mathrm{Mg}$ is critical for the development of new casting and wrought Mg alloys.

Fortunately, it is now possible to calculate many aspects of diffusion [13, 14]. Firstprinciples calculations based on density functional theory (DFT) have been extensively used to calculate diffusion coefficients, especially when experimental data are lacking $[15,16]$. These calculations are usually coupled with transition state theory (TST) under the harmonic or the quasi-harmonic approximations [14]. TST has become a practical tool in the context of DFT calculations when efficient algorithms for finding the minimum-energy path have been developed, such as the nudged elastic band (NEB) and the climb image nudged elastic band (CINEB) method [17]. At present, first-principles calculations of diffusion coefficients are largely limited to cubic systems, such as those in $\mathrm{Al}[15,18], \mathrm{Fe}[19,20]$, and $\mathrm{Ni}$ [21-23] alloys. This is due to the additional complexity of anisotropy associated with the calculations of diffusion coefficients in hep systems. Recently, Ganeshan et al. [16] in our group calculated the diffusion coefficients of $\mathrm{Al}, \mathrm{Zn}, \mathrm{Sn}$, and $\mathrm{Ca}$ in dilute hcp Mg using an 8-frequency model. However, their calculated results compared with experimental data still need to be further improved (see details in Section 4), and especially more alloying elements need to be considered for Mg alloys.

In the present study, we use first-principles calculations coupled with the TST and the 8frequency model to calculate the dilute solute tracer diffusion coefficients in hcp Mg. Fortyseven substitutional alloying elements have been considered herein, namely $\mathrm{Ag}, \mathrm{Al}, \mathrm{As}, \mathrm{Au}, \mathrm{Be}$, 
Bi, Ca, Cd, Co, Cr, Cu, Fe, Ga, Ge, Hf, Hg, In, Ir, Li, Mn, Mo, Na, Nb, Ni, Os, Pb, Pd, Pt, Re, Rh, Ru, Sb, Sc, Se, Si, Sn, Sr, Ta, Tc, Te, Ti, Tl, V, W, Y, Zn, and Zr (see also Figure 1). The self-diffusion coefficient of $\mathrm{Mg}$ has been calculated as well. The effects of different exchangecorrelation (X-C) functionals on diffusion properties are examined. It is shown that the recently developed PBEsol X-C functional [24] yields better agreement with experimental data compared with the commonly used X-C functionals such as the local density approximation (LDA) and the generalized gradient approximation (GGA) for the self-diffusion [25] and solute diffusion coefficients (Al, Sn, Zn) in Mg [16] calculated in previous works. The vibrational properties are derived from the quasi-harmonic Debye model $[26,27]$. Therefore, we are able to calculate not only the migration barriers but also the temperature-dependent jump frequencies and the diffusion pre-factors, which are related to vibrational entropic contributions. Finally the dilute solute tracer diffusion coefficients in hep $\mathrm{Mg}$ are calculated. The diffusion pre-factors and the activation energies are obtained by fitting the calculated diffusion coefficients to the Arrheniustype diffusion equation (see details in Section 2).

The remainder of this paper is organized as follows. In Section 2, a detailed description of diffusion theory in an hep lattice is provided, including an overview of diffusion equations used in hep system and the 8-frequency model to calculate the dilute solute tracer diffusion coefficients. The DFT methodology used to calculate the inputs for the diffusion equations and the 8-frequency model is detailed in Section 3. Calculation results are examined in Section 4, followed by a summary in Section 5 . 


\section{Atomic diffusion theory for hep lattice}

Note that solute diffusion in dilute alloys is also referred to impurity diffusion in the literature [28]. In the remainder of the paper, "solute" is synonymous with "impurity". For the hcp lattice, the crystal anisotropy results in two unique solute jumps, one within the basal plane and the other between adjacent basal planes. These two solute jumps in the hcp structure are illustrated in Figure 2. This leads to two distinctive diffusion tensors. The diffusion coefficient perpendicular to the $c$-axis, $D_{\perp}$, results from jumps between the adjacent basal planes and jumps within the basal plane; while the diffusion coefficient parallel to the $c$-axis, $D_{\square}$, attributes to jumps between the adjacent basal planes only. Correspondingly, the diffusion coefficients can be calculated using the following equations [29]:

$$
\begin{aligned}
& D_{\perp}=\frac{1}{2} C_{v} a^{2}\left(3 f_{B x} \omega_{X}+f_{A b} \omega_{X}^{\prime}\right) \\
& D_{\square}=\frac{3}{4} C_{v} c^{2} f_{A z} \omega_{X}^{\prime}
\end{aligned}
$$

where $a$ and $c$ are lattice parameters of hep lattice, $C_{v}$ corresponds to vacancy concentration adjacent to the solute atom, $\omega_{X}$ and $\omega_{X}^{\prime}$ are the solute-vacancy exchange jump frequencies within and out of the basal plane, respectively, $f_{B x}$ is the partial correlation factor for the solute jump within a basal plane, $f_{A b}$ is the partial correlation factor corresponding to the horizontal component of a solute jump into an adjacent basal plane, and $f_{A z}$ is the partial correlation factor corresponding to the vertical component of a solute jump into the adjacent basal plane. Details of the calculations of these variables are given below. 


\subsection{Vacancy concentration adjacent to a solute atom}

In order for a solute atom to jump, a vacancy adjacent to the solute atom needs to form. The vacancy concentration adjacent to the solute atom, $C_{v}$ in Eqs. (1) and (2) in the dilute limit, is calculated by a Boltzmann relation:

$$
C_{v}=\exp \left(-\frac{\Delta G_{f}}{k_{B} T}\right)
$$

where $\Delta G_{f}=\Delta G_{f}^{0}-\Delta G_{b}$ is the free energy of vacancy formation adjacent to the solute atom. $\Delta G_{f}^{0}$ is the vacancy formation free energy in pure $\mathrm{Mg}$ without solute, as is calculated by

$$
\Delta G_{f}^{0}=G\left(\mathrm{Mg}_{N-1} \mathrm{Va}_{1}\right)-\frac{N-1}{N} G\left(\mathrm{Mg}_{N}\right)
$$

where Va indicates a vacancy, and $N$ the number of lattice sites in the supercell. $\Delta G_{b}$ is the solute-vacancy binding free energy, defined as the free energy difference between a solute atom/vacancy pair and the two as isolated defects [30,31]:

$-\Delta G_{b}(\mathrm{X}-\mathrm{Va})=G\left(\mathrm{Mg}_{N-2} \mathrm{X}_{1} \mathrm{Va}_{1}\right)+G\left(\mathrm{Mg}_{N}\right)-G\left(\mathrm{Mg}_{N-1} \mathrm{X}_{1}\right)-G\left(\mathrm{Mg}_{N-1} \mathrm{Va}_{1}\right)$

where $\mathrm{X}$ represents a solute atom. The minus sign in front of $\Delta G_{b}$ in Eq. (5) is to keep the binding energy consistent with the convention in the literature such that favorable solute-vacancy binding is positive [32]. $\Delta G_{b}$ shows the effect of solute-vacancy interaction on the vacancy concentration adjacent to the solute atom. In the course of calculating solute-vacancy binding energy, the dilute mixing energy $E_{m i x}$ for $\mathrm{X}$ in hep Mg solid solution is readily calculated by:

$$
E_{m i x}(\mathrm{X})=E\left(\mathrm{Mg}_{\mathrm{N}-\mathrm{I}} \mathrm{X}_{1}\right)-\frac{N-1}{N} E\left(\mathrm{Mg}_{\mathrm{N}}\right)-E(\mathrm{X})
$$

where $E(\mathrm{X})$ is the total energy of the solute element in its ground state structure. 


\subsection{Jump frequencies of solutes}

Once a vacancy adjacent to a solute atom is formed, the solute atom has to overcome the migration barrier to exchange with the vacancy. Based on Eyring's reaction rate theory [33] of activated complex, as applied by Wert and Zener [34] to solute diffusion in solids, the solutevacancy exchange jump frequencies within and between the basal planes $\omega_{X}$ and $\omega_{X}^{\prime}$ in their general form can be written as:

$\omega=\frac{k_{B} T}{h} \exp \left(-\frac{\Delta G_{m}}{k_{B} T}\right)$

where $\Delta G_{m}=G_{T S}-G_{I S}$ is the solute migration free energy barrier with $G_{T S}$ being the free energy of the transition state (TS) and $G_{I S}$ the free energy of the initial state (IS). A detailed discussion of Eq. (7) at the high temperature and low temperature ranges is given by Wimmer et al. [35]. Instead of using Vineyard's harmonic TST $[19,36]$ which requires computation of full phonon frequencies, we calculate the jump frequency directly from free energy calculations using quasiharmonic Debye model as discussed in Section 3.3.

\subsection{Correlation factors and the 8-frequency model}

One solute atom can exchange positions with a vacancy and subsequently jump back to its original position. This results in zero net diffusion distance for the solute atom. The degree to which this effect hinders diffusion depends on the correlation between two successive solutevacancy exchanges, which can be described by a correlation factor $f$. The correlation factor is a function of solute and solvent jump frequencies and geometry of crystal lattice. For self-diffusion in hcp, vacancy jumps are almost equally probable, thus the correlation factor is mainly determined by the lattice geometry. The hcp self-diffusion correlation factors are temperature- 
dependent and can be calculated using the method suggested by Mullen [37]. The presence of a solute, however, can alter the vacancy migration by biasing certain jumps and thus the value of $f$ will change relative to that of self-diffusion.

The correlation factors of solute diffusion in an hep lattice can be quantitatively evaluated using the 8 -frequency model developed by Ghate [38], which is analogous to the 5-frequency model by Le Claire [39] for fcc materials. In Ghate's model these correlation factors depend on 8 jump frequencies illustrated in Figure 3 and are expressed as:

$$
\begin{aligned}
& f_{A z}=\frac{2 \omega_{a}^{\prime}+5.512 \omega_{c}^{\prime}}{2 \omega_{a}^{\prime}+5.512 \omega_{c}^{\prime}+2 \omega_{X}^{\prime}} \\
& f_{B x}=1+\frac{2 S_{B x}}{\lambda_{B}} \\
& f_{A b}=1+\frac{2 S_{A b}}{\lambda_{A b}}
\end{aligned}
$$

where jump distances $\lambda_{B}$ and $\lambda_{A b}$ correspond to the net jump distances associated with jumps within a basal plane and between adjacent basal planes, respectively (see Figure 2). The average final displacements $S_{B x}$ and $S_{A b}$ are determined by solving the following system of equations:

$$
\begin{aligned}
& S_{A b}=\frac{\omega_{a}^{\prime}\left(\sqrt{3} S_{B x}+S_{B y}\right)-\omega_{b}^{\prime} S_{A b}-\omega_{X}^{\prime}\left(\lambda_{A b}+S_{A b}\right)}{2 \omega_{a}^{\prime}+2 \omega_{b}^{\prime}+5.512 \omega_{c}^{\prime}+\omega_{X}^{\prime}} \\
& S_{B x}=\frac{\sqrt{3} \omega_{a} S_{A b}+\omega_{b} S_{B x}-\omega_{X}\left(\lambda_{B}+S_{B x}\right)}{2 \omega_{a}+2 \omega_{b}+5.512 \omega_{c}+\omega_{X}} \\
& S_{B y}=\frac{\omega_{a} S_{A b}-\omega_{b} S_{B y}}{2 \omega_{a}+2 \omega_{b}+5.512 \omega_{c}+\omega_{X}}
\end{aligned}
$$

Using the frequencies of each jump in the 8-frequency model, the Eqs. (11) (13) can be solved and the correlation factors can be calculated using Eqs. (8) (10). 
Free energies $\Delta G_{f}$ and $\Delta G_{m}$ can be obtained from DFT calculations based on the quasiharmonic approximations described in Section 3.3 to calculate the jump frequencies $\omega_{X}, \omega_{X}^{\prime}$, $\omega_{a}, \omega_{b}, \omega_{c}, \omega_{a}^{\prime}, \omega_{b}^{\prime}, \omega_{c}^{\prime}$, and the vacancy concentrations. We then implement them into the 8frequency model, and subsequently obtain the diffusion coefficients from Eqs. (1) and (2). The calculated diffusion coefficients $D$ in Eqs. (1) and (2) can be fitted into the conventional Arrhenius form:

$D=D_{0} \exp \left(-\frac{Q}{k_{B} T}\right)$

where $Q$ is the diffusion activation energy and $D_{0}$ the pre-exponential factor. The activation energy $Q$ corresponds to the enthalpy of vacancy formation, the enthalpy of solute migration, and part of the contribution from correlation factors; the diffusion pre-exponential factor $D_{0}$ corresponds to the entropy of vacancy formation, the entropy of solute migration, the lattice parameters, and also part of the correlation factors. It should be noted that all the diffusion plots shown in the present work were plotted using the calculated data directly from first-principles, not the fitted Arrhenius equation (see the calculated diffusion data set in the associated data article Ref [40]).

\section{Computational methods}

First-principles calculations based on DFT were employed to calculate the free energies needed in the diffusion equations and the 8-frequency model. The ion-electron interaction was described by the projector augmented plane-wave (PAW) method [41] and the X-C functional was described by an improved GGA of PBEsol [24], as implemented in the VASP 5.3.2 code [42]. The suitability of PBEsol for the present study is discussed in the Section 4. The 
recommended core configurations by VASP were adopted for each element in the present work, as shown in Figure 1. Due to the magnetic nature of V, Cr, Mn, Fe, Co, and Ni, first-principles calculations containing these elements were performed with the spin polarization approach. An energy cut-off of $350 \mathrm{eV}$ was used for the plane-wave expansion of the electronic wave functions.

\subsection{Supercell size and k-point density}

Tests were performed on pure $\mathrm{Mg}$ and representative solutes $\mathrm{Zn}$ and $\mathrm{Y}$ to estimate the convergence of the calculation results with respect to the supercell size and $k$-point density (see Section 2.1 and 2.2 in Ref [40]). On the basis of these tests, the vacancy formation, migration and binding energies are estimated to be converged to within a precision of approximately 0.01 $\mathrm{eV}$.

After tests of supercell size, we chose a 64 -atom supercell $(4 \times 4 \times 2$ conventional hcp unit cells) for most solutes, which was sufficient to isolate the vacancy and solute atom from the periodic images. For $\mathrm{Ba}, \mathrm{Bi}, \mathrm{Ca}, \mathrm{K}, \mathrm{Pb}, \mathrm{Sr}$, and $\mathrm{Y}$, a 96 -atom $4 \times 4 \times 3$ supercell was used due to the large atomic size of these elements. All structural degrees of freedom (ionic coordinates, cell volume, and cell shape) in the supercell were fully relaxed via a conjugate gradient method to an energy convergence of $10^{-5} \mathrm{eV} /$ atom, followed by a final static calculation using the tetrahedron method with Blöchl corrections [43] with energy convergence of $10^{-6} \mathrm{eV} /$ atom to get the accurate total energy. Although constant volume and cell shape were used in other diffusion calculations $[19,44,45]$, full relaxation of all degrees of freedom can result in more accurate local strain and generate the useful quantity $\Delta V_{X}$, which will be discussed in Section 4.2 . An $8 \times 8 \times 9 \Gamma$-centered $k$-point mesh was used for the 64-atom supercell for the electronic integration in the Brillouin 
zone. For calculations using 96 -atom supercells, a $5 \times 5 \times 4 \Gamma$-centered $k$-point mesh was used in structural relaxation and a $7 \times 7 \times 7 \Gamma$-centered $k$-point mesh in subsequent static calculations.

\subsection{Transition state search}

To determine the position of the saddle point and associated minimum-energy pathway during solute migration, calculations were performed employing the CI-NEB method [17] with 3 images. The two end-point structures, i.e. the initial and final structures, were fully relaxed first. The same supercell size and $k$-point settings were used in the CI-NEB calculations as are mentioned in Section 3.1. Such calculations were performed for all the solute (solvent)-vacancy exchange in the 8-frequency model for each investigated elements. Unlike hcp Ti (as well as hcp Zr) with two saddle points [46], in all cases the energy was found to display a single maximum, corresponding to the saddle point at the high-symmetry position located half way between neighboring sites.

\subsection{The quasi-harmonic approach}

Vibrational contributions to the free energies were calculated using the quasi-harmonic approximations from phonon or Debye model. Helmholtz free energy of a configuration as a function volume $(V)$ and temperature $(T)$ can be expressed as [27]:

$F(V, T)=E_{0}(V)+F_{v i b}(V, T)+F_{e l}(V, T)$

where $E_{0}(V)$ is the static energy at $0 \mathrm{~K}$ without the zero-point vibrational energy, $F_{v i b}(V, T)$ the vibrational contribution, and $F_{e l}(V, T)$ the thermal-electronic contribution. Note that at zero pressure, the Helmholtz free energy is equal to the Gibbs free energy. In the present work, $E_{0}(V)$ was calculated via first-principles directly, and $F_{e l}(V, T)$ obtained by integrating the 
electronic density of states [47]. It is found that $F_{e l}(V, T)$ has a negligible contribution to the total free energy compared with $F_{v i b}(V, T)$, but it was still considered in the present work for the sake of completeness. Based on the previous work [23] from our group, the quasi-harmonic Debye model is sufficient to describe well the vibrational contribution to free energy in diffusion calculations compared with phonon calculations and can save tremendous computing resources. Therefore, $F_{v i b}(V, T)$ for all the initial and transition states were calculated using the quasiharmonic Debye model in the present work. We still calculated the self-diffusion coefficients in Mg using quasi-harmonic phonon model for comparison with the quasi-harmonic Debye model, as shown in Figure 5.

In the quasi-harmonic phonon model, the vibrational contribution to Helmholtz energy is obtained by the integration of calculated phonon density of states. The details of implementation can be found in our previous works [27, 47]. In the quasi-harmonic Debye model [26, 27], the vibrational contribution to Helmholtz energy is described as

$F_{v i b}(V, T)=\frac{9}{8} k_{B} \Theta_{D}(V)-k_{B} T\left[D\left(\frac{\Theta_{D}(V)}{T}\right)+3 \ln \left(1-e^{-\frac{\Theta_{D}(T)}{T}}\right)\right]$

where $\Theta_{D}$ is the Debye temperature, $\frac{9}{8} k_{B} \Theta_{D}(V)$ the zero-point vibrational energy, and $k_{B}$ the Boltzmann's constant. The Debye function, $D(x)$, is defined as $D(x)=\frac{3}{x^{3}} \int_{0}^{x} \frac{z^{3} d z}{e^{z}-1}$.

To solve Eq. (16), the Debye temperature $\Theta_{D}$ must be calculated. In the present work, the Debye-Grüneisen approximation (Moruzzi-Janak-Schwarz approximation) [48] is used to describe $\Theta_{D}$ : 
$\Theta_{D}=s A V_{0}^{1 / 6}\left(\frac{B_{0}}{M}\right)^{1 / 2}\left(\frac{V_{0}}{V}\right)^{\gamma}$

where $A$ is a constant, representing $\left(6 \pi^{2}\right)^{1 / 3} h / k_{B}, V_{0}$ the ground state equilibrium volume, $M$ the average atomic mass, $\gamma$ the Grüneisen parameter, $B_{0}$ the bulk modulus, and $s$ a parameter that scales the Debye temperature and depends on the Poisson's ratio of materials [49]. $s=0.69$ was used in the present work to benchmark the Debye temperature of pure $\mathrm{Mg}(330 \mathrm{~K})$ obtained by the quasi-harmonic phonon calculations. Excellent agreement was obtained between the thermodynamic properties (heat capacity $\pm 1 \mathrm{~J} / \mathrm{mol}$, entropy $\pm 0.5 \mathrm{~J} / \mathrm{mol} / \mathrm{K}$ ) calculated from the quasi-harmonic phonon and Debye model in pure $\mathrm{Mg}$ (see Section 2.3 in Ref [40]).

Equilibrium properties for input in the Debye model, including volume $\left(V_{0}\right)$, energy $\left(E_{0}\right)$, bulk modulus $\left(B_{0}\right)$ and its pressure derivative $\left(B_{0}{ }^{\prime}\right)$, were obtained from an energy-volume equation of state (EOS) calculated from first-principles using the equilibrium volume $V_{0}$ and at least five additional volumes $\left(0.96,0.98,1.02,1.04\right.$, and 1.06 with respect to $\left.V_{0}\right)$. A four parameter Birch-Murnaghan EOS was used herein to fit the energy-volume data. It is represented in its linear form [27]

$E(V)=a+b V^{-2 / 3}+c V^{-4 / 3}+d V^{-2}$

where $a, b, c$, and $d$ are fitting parameters. This EOS was adopted because it has been previously shown to be able to produce accurate properties for many materials systems $[27,50]$. It should be noted that the EOS for the saddle point configuration was obtained without relaxing each additional volume in order to maintain its saddle point structure. It is observed that the value of $B_{0}$ ' is quite sensitive to the EOS fitting and is always around 4 in all the EOS fittings (see the associated diffusion data file in Ref [40]). When the fitted energy-volume curve was not smooth 
enough due to the scattered energy-volume points which indicates the $B_{0}$ ' value is far away from 4, the value from a pressure-volume fitting [51] was used instead.

\section{Results and Discussion}

\subsection{Vacancy formation energy in $\mathrm{Mg}$}

Vacancy formation energy $\Delta E_{f}^{0}$ is critical for obtaining accurate diffusion coefficients because it is exponentially related to the vacancy concentration in pure $\mathrm{Mg}$, which is a term presented in all the vacancy-mediated diffusion equations in the present work. Previously measured [52-58] and calculated [14, 25, 30, 59, 60] values for $\Delta E_{f}^{0}$ in $\mathrm{Mg}$ are listed Table 1 along with our present calculations and are in good agreement. Beevers [52], Mairy et al. [53], and Tzanétakis et al. $[55,58]$ measured $\Delta E_{f}^{0}$ in $\mathrm{Mg}$ using electrical resistivity method, with their measured values ranging from $0.79 \mathrm{eV}$ to $0.89 \mathrm{eV}$. Janot et al. [54] measured $\Delta E_{f}^{0}$ in $\mathrm{Mg}$ using the differential dilatometry and obtained $0.58 \mathrm{eV}$. The work of Janot et al. [54] was subsequently criticized by Tzanétakis et al. [55] and Hood [61] for suffering large uncertain errors in the thermal dilatometry measurements. Segers et al. [56] and Hautojärvi et al. [57] performed positron annihilation spectroscopy measurements of $\Delta E_{f}^{0}$ in $\mathrm{Mg}$ and obtained consistent values of around $0.85 \mathrm{eV}$. The vacancy formation entropy was also estimated to be $2 k_{\mathrm{B}}$ in the work of Hautojärvi et al. [57]. Our DFT calculated vacancy formation enthalpy and entropy from PBEsol compare especially well with the more reliable positron annihilation spectroscopy data $[56,57]$ (see Section 2.4 in $\operatorname{Ref}[40]$ ).

The introduction of vacancy into a system creates a small amount of internal surface inside the supercell. It has been indicated in several studies $[62,63]$ on vacancy formation in 
metallic systems that LDA will describe the internal surface around a vacancy better than GGA due to an error cancellation effect: LDA largely overestimates the exchange energy of a free metal surface, but underestimates by approximately the same magnitude the correlation energy. This results in a reasonable net total value of the surface energy [64]. In the present work, we applied the X-C functional of PBEsol because it includes the surface correction based on a gradient expansion of the exchange energy and a final fit of the X-C energy to that of surface jellium [24]. Therefore it is able to describe well the internal surface of the vacancy. Consequently, the vacancy formation energy values calculated from LDA and PBEsol agree very well with those measured by the positron annihilation spectroscopy in the literature, which is around $0.85 \mathrm{eV}$ for $\mathrm{Mg}$ [61]. GGA systematically underestimates the vacancy formation energy by around $0.1 \mathrm{eV}$, as is shown by two of our previous studies $[25,30]$ listed in Table 1 .

We also tested the effect of supercell size on the calculated vacancy formation energy and found that 64 -atom supercell is sufficient to converge the result. Using the vacancy formation energy and entropy in pure hcp $\mathrm{Mg}$ calculated from PBEsol ( $0.86 \mathrm{eV}$ and $2.1 k_{\mathrm{B}}$, respectively) the equilibrium vacancy concentration at $920 \mathrm{~K}$ near the melting point of $\mathrm{Mg}$ is calculated to be a reasonable value of $1.5 \times 10^{-4}$ according to Eq. (3). Recently, Glensk et al. [65] have demonstrated that the traditional linear Arrhenius assumption to extrapolate the experimental $\mathrm{T}=0 \mathrm{~K}$ enthalpy of vacancy formation is not accurate using $\mathrm{Al}$ and $\mathrm{Cu}$ as examples. They proposed that linear Arrhenius assumption needs to be replaced by a local Grüneisen theory with a formation entropy linear in the temperature by considering the anharmonic contribution to the vibrational free energy. However, their proposed theory needs to be further validated in more materials systems. 


\subsection{Solute-vacancy binding energy}

The binding energies of solute-vacancy pairs in Mg modify the vacancy concentration adjacent to the solute atom and have been reported in Table 2. The present DFT results show a good agreement with those calculated by Shin et al. [30] using GGA as the X-C functional. A positive sign on the solute-vacancy binding energies indicates favorable binding (attractive) while negative sign indicates unfavorable binding (repulsive). Vydyanath et al. [66] and Rao and Suryanarayana [67] measured the vacancy-binding energies of $\mathrm{Zn}$ and $\mathrm{Al}$ in $\mathrm{Mg}$, respectively, both from quenching experiments as shown in Table 2. There is a large discrepancy between the measured $0.29 \mathrm{eV}$ [67] and the calculated $0.02 \mathrm{eV}$ for Al-vacancy binding in $\mathrm{Mg}$, which may be due to inaccuracies of non-equilibrium measurements. The measured Zn-vacancy binding energy of $0.07 \mathrm{eV}$ in $\mathrm{Mg}$ [66] is in good agreement with the calculated $0.05 \mathrm{eV}$ in the present work.

For a quantitative measure of the atomic size of each solute $\mathrm{X}$, the volume difference $\Delta V_{X}$ induced by placing a single solute into pure $\mathrm{Mg}$ is calculated and shown in Table 2, which is expressed as [45]:

$\Delta V_{X}=V\left(\operatorname{Mg}_{N-1} \mathrm{X}_{1}\right)-V\left(\mathrm{Mg}_{N}\right)$

Except for transition metals (TMs), modest correlation is found between solute-vacancy binding energy and $\Delta V_{X}$. This correlation can be explained by a simple strain model proposed by Shin [30]. It states that a large solute atom in Mg will induce a significant strain on the surrounding $\mathrm{Mg}$ atoms, and a vacancy adjacent to such a large solute atom will allow the solute atom to relax towards the vacancy and relieve the strain. Therefore for solutes with large atomic size, large solute-vacancy binding energies are obtained due to the large induced strain, such as $\operatorname{Sr}(0.30$ $\mathrm{eV}), \mathrm{K}(0.40 \mathrm{eV})$, and $\mathrm{Ba}(0.67 \mathrm{eV})$. 
However, this strain model does not explain the binding behavior of TMs in Mg. Figure 4 shows the solute-vacancy binding energies of various solutes within the basal plane. Early TMs, e.g. Ti, $\mathrm{V}, \mathrm{Cr}$, and $\mathrm{Mn}$ for $3 d$ metals, have unfavorable binding with vacancy in $\mathrm{Mg}$, while late TMs, e.g. $\mathrm{Fe}, \mathrm{Co}, \mathrm{Ni}, \mathrm{Cu}$, and $\mathrm{Zn}$ for $3 d$ metals, have favorable binding with vacancy in $\mathrm{Mg}$. The solute-vacancy binding energies follow the similar trend as a function of atomic number for $3 d$, $4 d$, and $5 d$ TMs, exhibiting a flatwise "S" shape curve in Figure 4. Simonovic and Sluiter [44] also found that TMs in Al bind with vacancies in a similar manner.

Solutes with strong vacancy binding might strongly affect the precipitation behavior of other alloying elements because of vacancy trapping after the solutionizing heat treatment [3]. For example, the very strong vacancy binding of Sr causes a significant decrease of the vacancy formation energy adjacent to a Sr solute and may result in vacancy trapping. However, the MgSr system has very positive enthalpy of mixing in the hcp phase [68], causing Sr to be repelled from $\mathrm{Mg}$ atoms. Therefore, the $\mathrm{Sr}$ atom forms a complex with a vacancy in which the $\mathrm{Sr}$ atom is displaced toward the vacancy to relieve the strain. The barrier for Sr-vacancy exchange $E_{X}$ is extremely low $(0.01 \mathrm{eV})$ because the $\mathrm{Sr}$ atom sits almost at the midpoint between two sites. The same situation also applies to $\mathrm{K}$ and $\mathrm{Ba}$, for which the solute-vacancy binding becomes so strong that their migration barriers are vanishingly small.

\subsection{Diffusion data}

\subsubsection{Effects of X-C functionals}

Figure 5 shows the calculated $\mathrm{Mg}$ self-diffusion coefficients using different $\mathrm{X}-\mathrm{C}$ functionals of LDA, GGA, and PBEsol compared with available experiments [69-72]. The selfdiffusion coefficients from PBEsol calculated by Debye model show the best agreement with 
available experimental values. According to the Arrhenius diffusion of Eq. (14), the slope of the diffusion coefficient line in Figure 5 corresponds to the activation energy $Q$ and the intersect with vertical axis corresponds to the diffusion pre-factor $D_{0}$. The enthalpies of vacancy formation and migration mainly contribute to $Q$ while the entropies of vacancy formation and migration mainly contribute to $D_{0}$.

Diffusion coefficients calculated using LDA yield a decent slope but a lower intersect, while the calculations using GGA give a good intersect but a lower slope compared with experiments. The reason is because LDA and GGA have different advantages in terms of getting accurate properties. LDA is most suitable for vacancy formation energy due to its surface error cancellation [73] as discussed in Section 4.1. But LDA causes significant overbinding in metallic systems, evidenced by the underestimated $\mathrm{Mg}$ equilibrium lattice parameters $\left(a_{0}=3.112\right.$ [59], 3.12 [60], 3.141 [14], $3.133 \AA$ [25] and $c_{0}=5.041$ [59], 5.06 [60], 5.065 [14], $5.079 \AA$ [25]) compared with experiment $\left(a_{0}=3.209 \AA\right.$ and $c_{0}=5.211 \AA$ [74]) in Table 1, which results in a lower $D_{0}$. On the contrary, although improper for vacancy formation energy, GGA is most suitable to obtain the equilibrium and vibrational properties for metallic systems [50]. This explains why the self-diffusion coefficients from LDA have a decent slope, i.e. the enthalpy-related $Q$ value, and a good intersect from GGA, i.e. the entropy-related $D_{0}$ value. Since PBEsol is a revised GGA that improves equilibrium properties of densely packed solids and their surfaces [24], and possesses both advantages from LDA and GGA, it hence improves the predictions for both $Q$ and $D_{0}$.

\subsubsection{Comparison between Debye and phonon model}

As shown in Figure 5, the calculated self-diffusion coefficients by the quasi-harmonic Debye model shows better agreement with experiments than the quasi-harmonic phonon model. 
The Debye and phonon models yield different entropies of vacancy formation and entropies of vacancy migration, which attributes to the intersect difference $\left(D_{0}\right)$ between the self-diffusion coefficients calculated by these two models. The Debye model has more accurate intersect compared with experiments while the phonon model underestimates the $D_{0}$ value by around half an order of magnitude. Debye model is expected to perform well in systems where the differences in vibrational free energy between structures can be explained by uniform shifts of the phonon DOS, such as when the volume effect operates alone [75]. In the calculations of diffusion coefficients in $\mathrm{Mg}$, the major contribution to the entropic differences between different supercell configurations is the solute size effect, as represented by the solute-induced volume change $\Delta V_{X}$. This is the reason for the success of Debye model. The present work shows that the Debye model is a valid alternative for the calculations of diffusion coefficients that will reduce computational time and complexity of the calculations. Similar conclusion has been drawn in a recent work on the calculation of self-diffusion coefficients in fcc Ni [23].

\subsubsection{Correlation effects}

From Eqs. (1) and (2), it is seen that the diffusion coefficients are linearly dependent on the correlation factors, which can range from 0 to 1 . If correlation effects are weak, the correlation factors can be ignored because their values are close to 1 . They will have little effects on the final calculated diffusion coefficients, as shown by Ganeshan et al. [16] for Al, Zn, and Sn diffusions in Mg. However, if the correlation effects are strong, the correlation factors become small and greatly decrease the calculated diffusion coefficients. For examples, the calculated correlation factors of $\mathrm{Ca}$ and $\mathrm{Zn}$ diffusion in $\mathrm{Mg}$ as a function of temperature are shown in Figure 6(b). Correlation factors of $\mathrm{Zn}$ are all above 0.9 , while correlation factors of $\mathrm{Ca}$ are all 
below 0.25 . Interestingly, the correlation factors of $\mathrm{Ca}$ increases as the temperature increases while those of $\mathrm{Zn}$ show the opposite trend. Figure 6(a) shows the calculated diffusion coefficients of $\mathrm{Ca}$ and $\mathrm{Zn}$ in $\mathrm{Mg}$ with and without correlation effects considered. Diffusion coefficients of $\mathrm{Ca}$ with and without correlation differ around one order of magnitude, showing non-negligible effects of correlation. Diffusion coefficients of $\mathrm{Zn}$ with and without correlation almost overlap with each other in Figure 6(a), showing little effects of correlation. More diffusion plots showing comparisons with and without correlation effects considered can be found in Section 3.1 and 3.2 in Ref [40].

The correlation effects can be further understood by the migration barriers of each jump in the 8 -frequency model. Table 3 lists the energy barriers of vacancy migration for solutes $\mathrm{Zn}$, $\mathrm{Pb}, \mathrm{Bi}, \mathrm{Ca}, \mathrm{Na}, \mathrm{Se}, \mathrm{Sr}, \mathrm{Te}$, and $\mathrm{Y}$ in $\mathrm{Mg}$. As shown in Table 3, the direct solute migration barriers $\left(E_{X}\right.$ and $\left.E_{X}^{\prime}\right)$ for $\mathrm{Zn}$ and $\mathrm{Pb}$ are larger than the other solvent $(\mathrm{Mg})$ migration barriers $\left(E_{a}, E_{b}, E_{c}\right.$, $E_{a}^{\prime}, E_{b}^{\prime}$, and $E_{c}^{\prime}$ ). While for the elements with strong correlation effects, i.e. $\mathrm{Ca}, \mathrm{Sr}, \mathrm{Y}, \mathrm{Na}, \mathrm{Se}$, and $\mathrm{Te}$, the direct solute migration barriers are significantly smaller than the solvent migration barriers. For example, the direct $\mathrm{Sr}$ migration barriers, $E_{X}=0.01 \mathrm{eV}$ and $E_{X}^{\prime}=0.02 \mathrm{eV}$, are extremely small due to the strong solute-vacancy binding mentioned in Section 4.2. The small direct solute migration barriers lead to fast solute-vacancy oscillations which do not themselves contribute to the net diffusion. This large difference between the direct solute migration barriers and the solvent migration barriers is the origin of the strong correlation effects of these solutes. The elements with much bigger direct solute migration barriers than the solvent migration barriers, such as the transition metals, have very weak correlation effects. Therefore, the six solvent $(\mathrm{Mg})$ atoms jump frequencies $\left(\omega_{a}, \omega_{b}, \omega_{c}, \omega_{a}^{\prime}, \omega_{b}^{\prime}, \omega_{c}^{\prime}\right)$ for elements other than $\mathrm{Ca}, \mathrm{Na}$, $\mathrm{Se}, \mathrm{Sr}, \mathrm{Te}$, and $\mathrm{Y}$ were not calculated in this work because they don't contribute to the final 
diffusion coefficients significantly. For $\mathrm{Bi}$, it behaves almost like $\mathrm{Mg}$ self-diffusion because all of the migration barriers are close to $0.44 \mathrm{eV}$. The present study also confirms that 8 -frequency model is sufficient to capture the correlation effects of solute diffusion in hcp $\mathrm{Mg}$ in a quantitative level.

A very good indicator of the correlation effects is the volume difference $\Delta V_{X}$ induced by placing a single solute into pure $\mathrm{Mg}$ as discussed by Huber et al. [45]. Solutes with small or negative $\Delta V_{X}$, like $\Delta V_{Z n}=-11.1 \AA^{3} /$ supercell, show weak correlation effects, indicating the correlation factors for the diffusion of these solutes in $\mathrm{Mg}$ to be very close to 1 . Solutes with large positive $\Delta V_{X}$ such as $\mathrm{Ca}, \mathrm{Na}, \mathrm{Sr}, \mathrm{Te}$, and $\mathrm{Y}$ show strong correlation effects in diffusion, indicating the correlation factors in these systems to be much less than 1 and showing nonnegligible effects on the calculated diffusion coefficients. The effects of $\Delta V_{X}$ can be traced back to their migration barriers. Large atomic size induces large lattice misfit strain and causes large positive $\Delta V_{X}$, which leads to more local free space and thus lower $E_{X}$ for solutes like $\mathrm{Ca}, \mathrm{Na}, \mathrm{Sr}$, Te, and $\mathrm{Y}$, as shown in Figure 7. This indicates that the larger solute atoms can move faster while the smaller ones move slower. Interestingly, Se is an exception. Even with a negative $\Delta V_{X}$ $\left(-4.6 \AA^{3}\right)$, the migration barriers of Se are very small (e.g. $\left.E_{X}=0.08 \mathrm{eV}\right)$. This may be due to the fact that, unlike those solutes with large atomic size, the atomic size of Se $(1.03 \AA)$ is the smallest among all the solutes studied herein and is much smaller than that of $\operatorname{Mg}(1.45 \AA)$, which can also give Se more free space to move in the Mg lattice. 


\subsubsection{Bonding and trends in calculated diffusion data}

Table 4 summarizes the calculated diffusion coefficients for various solutes in $\mathrm{Mg}$ compared with available experiments [76-88]. In many experiments, the measured diffusion coefficient data points were few and scattered, and the fitted values of activation energy $Q$ and diffusion pre-factor $D_{0}$ have relatively large error bars. For solutes with more measurements available in the literature, such as $\mathrm{Al}[83-85,88], \mathrm{Zn}[77,82,86,88]$, and $\mathrm{Sn}$ [79], the present predictions agree remarkably well with experimental values within a factor of 3 , as shown in Figure 8. Solute diffusion results of Al, Zn, and Sn from LDA [16] show slopes compare well with experiments but consistently underestimate intersects of diffusion coefficients $\left(D_{0}\right)$, as seen in Table 1 and Figure 8. This can be understood by similar analysis discussed in Section 4.3.1 for Mg self-diffusion due to the overbinding of LDA. Figure 9 shows all of the basal dilute solute tracer diffusion coefficients in hcp $\mathrm{Mg}$ calculated in the present work. A large spectrum of values of diffusion coefficients across more than 10 orders of magnitude is found in solute diffusion in hcp Mg. More calculated diffusion coefficients compared with available experiments can be found in Section 3 in Ref [40].

As can be seen in Figure 10, the activation energy vs. atomic number curves show an inverse "V" shape, indicating the TM solutes have large activation energy. This may due to the strong interaction between solute atoms with $d$ electrons and the $\mathrm{Mg}$ matrix, which is traceable from the larger bulk moduli of these metals (see Table 2 and Figure 11). In an effort to seek the diffusivity trend, Figure 11 shows the basal diffusion coefficients of various solutes at $800 \mathrm{~K}$ as a function of bulk modulus for the $\mathrm{Mg}_{63} \mathrm{X}$ alloys $\left(\mathrm{Mg}_{95} \mathrm{X}\right.$ for $\mathrm{Bi}, \mathrm{Ca}, \mathrm{Pb}, \mathrm{Sr}$, and $\left.\mathrm{Y}\right)$. Bulk modulus represents the bonding strength between the solute atom and $\mathrm{Mg}$ matrix. Higher bulk modulus indicates stronger bonding between solute atom and $\mathrm{Mg}$ matrix and less mobile solute atom in 
the $\mathrm{Mg}$ matrix, resulting in a decreasing diffusion coefficient as the value of bulk modulus increases. In addition, the smaller solute atoms such as Re and W exhibit lower diffusivities in Mg due to their larger bulk moduli (see Figure 11). These observations are consistent with the conclusion drawn for solute diffusion in Ni-based alloys [21]. Most early TMs, such as Ti, V, Cr, $\mathrm{Mn}, \mathrm{Fe}, \mathrm{Ni}, \mathrm{Zr}$, and $\mathrm{Hf}$, do not form favorable bonds with $\mathrm{Mg}$. In binary $\mathrm{Mg}-\mathrm{X}$ alloy systems with these elements, no ordered intermetallic compounds are experimentally observed. These alloying elements in hcp $\mathrm{Mg}$ also tend to have positive enthalpies of mixing $\left(E_{m i x}>0\right)$, often indicating an energetic preference for phase separation and limited solid solubility, as shown in Table 2.

As, $\mathrm{Ca}, \mathrm{Ga}, \mathrm{Hg}, \mathrm{Li}, \mathrm{Na}, \mathrm{Sb}, \mathrm{Se}, \mathrm{Sr}, \mathrm{Te}, \mathrm{Tl}$, and $\mathrm{Zn}$ are faster diffusers than Mg selfdiffusion, while other solutes diffuse slower than Mg self-diffusion. Solutes which have high diffusion coefficients and low solid solubility, such as $\mathrm{Ca}, \mathrm{Na}, \mathrm{Sb}$, and $\mathrm{Sr}$, may serve as good alloying candidates for texture refinement since they have a high tendency to segregate to the grain boundaries and pin the grain boundary movement.

Although solute diffusion in $\mathrm{Mg}$ is anisotropic, the difference between values of $D_{\perp}$ and $D_{\square}$ is usually not very large since atomic migration is a local phenomenon. The ratio $D_{\perp} / D_{\square}$ is within a factor of 5 for most solutes, as shown in Figure 12. Note also that of all the experimentally measured solute diffusion coefficients, $D_{\perp}$ is larger than $D_{\square}$, with the exception being for Ag diffusion in $\mathrm{Mg}$. The diffusion coefficient $D_{\perp}$ of $\mathrm{Ag}$ in $\mathrm{Mg}$ is smaller than $D_{\square}$ from the experiments by Combronde and Brebec [79] (see Figure 4 in Ref [40]). The calculated diffusion coefficients differ with experimental data by one order of magnitude. Further experimental and computational work need to be done to elucidate this discrepancy. Meanwhile, for big solute atoms like $\mathrm{Ba}$ and $\mathrm{K}$, the $\mathrm{CI}-\mathrm{NEB}$ predicts that the saddle point configuration 
between the solute and vacancy sites is more stable than the initial configurations. Thus the diffusion coefficients in $\mathrm{Mg}$ for large solutes, $\mathrm{Ba}, \mathrm{K}, \mathrm{Rb}$, and $\mathrm{Cs}$ could not be calculated in terms of transition state theory.

\section{Summary}

As an effort for computational and data-driven development of advanced Mg-based alloys, we present a comprehensive study of dilute solute diffusion coefficients of 47 substitutional alloying elements in hcp Mg. The 8-frequency model for solute diffusion within an hcp lattice has been used to compute the correlation factors for different jumps. All the energies and vibrational quantities required to compute the jump frequencies and hence derive the solute diffusion coefficients from the 8-frequency model are calculated with the DFT based firstprinciples calculations in terms of the X-C functional of PBEsol and the quasi-harmonic Debye model. Saddle point configurations are predicted using the first-principles based CI-NEB method. It is found that:

(1) Compared with LDA and GGA, PBEsol is able to well describe both vacancy formation energies and vibrational properties, resulting in more accurate quantitative predictions of diffusion coefficients.

(2) Correlation effects are not negligible in solutes $\mathrm{Ca}, \mathrm{Na}, \mathrm{Se}, \mathrm{Sr}, \mathrm{Te}$, and $\mathrm{Y}$, in which the direct solute migration barriers are significantly smaller than the solvent migration barriers. This indicates that the larger solute atoms can move faster while the smaller ones move slower, with the exception of Se.

(3) The solute diffusion coefficients in hcp Mg are roughly inversely proportional to bulk modulus, i.e. their bonding to $\mathrm{Mg}$. Transition metal elements with $d$ electrons strongly 
interact with $\mathrm{Mg}$ and have very large diffusion activation energies, which cannot be explained by a simple strain model based on atomic size.

(4) The predicted solute diffusion coefficients in Mg compare remarkably well with available experiments in the literature. For most solutes basal diffusion coefficients are bigger than the non-basal ones. The diffusion coefficient of Ag shows anomaly with basal and nonbasal diffusion coefficients showing opposite trend compared with other alloying elements.

(5) The present work lays the foundation of diffusion data for future rational design of novel casting and wrought $\mathrm{Mg}$ alloys. The calculated diffusion data can be used to develop CALPHAD-type diffusion mobility databases for multi-component Mg alloys [89]. The theoretical methodology used herein can be readily applied to solute diffusion in other hcp systems.

\section{Acknowledgements}

The present work was funded by the National Science Foundation (NSF) through Grant No. DMR-1006557. First-principles calculations were carried out partially on the LION clusters at the Pennsylvania State University supported by the Materials Simulation Center and the Research Computing and Cyberinfrastructure unit at the Pennsylvania State University, partially on the resources of NERSC supported by the Office of Science of the US Department of Energy under contract No. DE-AC02-05CH11231, and partially on the resources of XSEDE supported by NSF with Grant No. ACI-1053575. Calculations were also carried out on the CyberSTAR cluster funded by NSF through Grant No. OCI-0821527. B.C.Z would like to thank Dr. Huazhi Fang for his helpful discussion. 


\section{References}

[1] B.L. Mordike, T. Ebert. Magnesium - properties - applications - potential, Mater. Sci. Eng. A 302 (2001) 37-45.

[2] T.M. Pollock. Weight loss with magnesium alloys, Science 328 (2010) 986-987.

[3] J.F. Nie. Precipitation and hardening in magnesium alloys, Metall. Mater. Trans. A 43A (2012) 38913939.

[4] S.L. Shang, H. Zhang, S. Ganeshan, Z.K. Liu. The development and application of a thermodynamic database for magnesium alloys, JOM 60 (2008) 45-47.

[5] G. Neumann, C. Tuijn. Self-diffusion and impurity diffusion in pure metals: handbook of experimental data, 2009.

[6] A.R. Antoniswarny, E.M. Taleff, L.G. Hector, J.T. Carter. Plastic deformation and ductility of magnesium AZ31B-H24 alloy sheet from 22 to $450^{\circ}$ C, Mater. Sci. Eng. A 631 (2015) 1-9.

[7] W.A. Curtin, D.L. Olmsted, L.G. Hector. A predictive mechanism for dynamic strain ageing in aluminium-magnesium alloys, Nature Materials 5 (2006) 875-880.

[8] J.A. Yasi, L.G. Hector, D.R. Trinkle. First-principles data for solid-solution strengthening of magnesium: From geometry and chemistry to properties, Acta Mater. 58 (2010) 5704-5713.

[9] K. Hono, C.L. Mendis, T.T. Sasaki, K. Oh-ishi. Towards the development of heat-treatable highstrength wrought Mg alloys, Scripta Mater. 63 (2010) 710-715.

[10] J.D. Robson. Effect of rare-earth additions on the texture of wrought magnesium alloys: The role of grain boundary segregation, Metall. Mater. Trans. A 45A (2014) 3205-3212.

[11] J.W. Cahn. The impurity-drag effect in grain boundary motion, Acta Metall. 10 (1962) 789-798.

[12] J.Y. Min, L.G. Hector, J.P. Lin, J.T. Carter, A.K. Sachdev. Spatio-temporal characteristics of propagative plastic instabilities in a rare earth containing magnesium alloy, Int. J. Plast. 57 (2014) $52-76$

[13] W.Y. Wang, B.C. Zhou, J.J. Han, H.Z. Fang, S.L. Shang, Y. Wang, X. Hui, Z.K. Liu. Prediction of diffusion coefficients in liquids and solids, Defect and Diffusion Forum 364 (2015) 182-191.

[14] M. Mantina, L.Q. Chen, Z.K. Liu. Predicting diffusion coefficients from first principles via Eyring's reaction rate theory, Defect and Diffusion Forum 294 (2009) 1-13.

[15] M. Mantina, Y. Wang, L.Q. Chen, Z.K. Liu, C. Wolverton. First principles impurity diffusion coefficients, Acta Mater. 57 (2009) 4102-4108.

[16] S. Ganeshan, L.G. Hector, Z.K. Liu. First-principles calculations of impurity diffusion coefficients in dilute Mg alloys using the 8-frequency model, Acta Mater. 59 (2011) 3214-3228.

[17] G. Henkelman, B.P. Uberuaga, H. Jonsson. A climbing image nudged elastic band method for finding saddle points and minimum energy paths, J. Chem. Phys. 113 (2000) 9901-9904.

[18] M. Mantina, Y. Wang, R. Arroyave, L.Q. Chen, Z.K. Liu, C. Wolverton. First-principles calculation of self-diffusion coefficients, Phys. Rev. Lett. 100 (2008) 215901.

[19] S.Y. Huang, D.L. Worthington, M. Asta, V. Ozolins, G. Ghosh, P.K. Liaw. Calculation of impurity diffusivities in $\alpha$-Fe using first-principles methods, Acta Mater. 58 (2010) 1982-1993.

[20] H. Ding, S.Y. Huang, G. Ghosh, P.K. Liaw, M. Asta. A computational study of impurity diffusivities for 5d transition metal solutes in alpha-Fe, Scripta Mater. 67 (2012) 732-735.

[21] A. Janotti, M. Krčmar, C.L. Fu, R.C. Reed. Solute diffusion in metals: larger atoms can move faster, Phys. Rev. Lett. 92 (2004) 085901.

[22] H.Z. Fang, S.L. Shang, Y. Wang, Z.K. Liu, D. Alfonso, D.E. Alman, Y.K. Shin, C.Y. Zou, A.C.T. van Duin, K. Lei, G.F. Wang. First-principles studies on vacancy-modified interstitial diffusion mechanism of oxygen in nickel, associated with large-scale atomic simulation techniques, J. Appl. Phys. 115 (2014) 043501.

[23] C.Z. Hargather, S.L. Shang, Z.K. Liu, Y. Du. A first-principles study of self-diffusion coefficients of fcc Ni, Comput. Mater. Sci. 86 (2014) 17-23. 
[24] J.P. Perdew, A. Ruzsinszky, G.I. Csonka, O.A. Vydrov, G.E. Scuseria, L.A. Constantin, X.L. Zhou, $\mathrm{K}$. Burke. Restoring the density-gradient expansion for exchange in solids and surfaces, Phys. Rev. Lett. 100 (2008) 136406.

[25] S. Ganeshan, L.G. Hector, Z.K. Liu. First-principles study of self-diffusion in hep Mg and Zn, Comput. Mater. Sci. 50 (2010) 301-307.

[26] M.A. Blanco, E. Francisco, V. Luana. GIBBS: isothermal-isobaric thermodynamics of solids from energy curves using a quasi-harmonic Debye model, Comput. Phys. Commun. 158 (2004) 57-72.

[27] S.L. Shang, Y. Wang, D. Kim, Z.K. Liu. First-principles thermodynamics from phonon and Debye model: Application to Ni and Ni3Al, Comput. Mater. Sci. 47 (2010) 1040-1048.

[28] P.G. Shewmon. Diffusion in solids, McGraw-Hill New York, 1963.

[29] N.L. Peterson. Self-diffusion in pure metals, J. Nucl. Mater. 69 (1978) 3-37.

[30] D. Shin, C. Wolverton. First-principles study of solute-vacancy binding in magnesium, Acta Mater. 58 (2010) 531-540.

[31] J.E. Saal, C. Wolverton. Solute-vacancy binding of the rare earths in magnesium from first principles, Acta Mater. 60 (2012) 5151-5159.

[32] C. Wolverton. Solute-vacancy binding in aluminum, Acta Mater. 55 (2007) 5867-5872.

[33] H. Eyring. The activated complex in chemical reactions, J. Chem. Phys. 3 (1935) 107-115.

[34] C. Wert, C. Zener. Interstitial atomic diffusion coefficients, Phys. Rev. 76 (1949) 1169-1175.

[35] E. Wimmer, W. Wolf, J. Sticht, P. Saxe, C.B. Geller, R. Najafabadi, G.A. Young. Temperaturedependent diffusion coefficients from ab initio computations: Hydrogen, deuterium, and tritium in nickel, Phys. Rev. B 77 (2008) 134305.

[36] G.H. Vineyard. Frequency factors and isotope effects in solid state rate processes, J. Phys. Chem. Solids 3 (1957) 121-127.

[37] J.G. Mullen. Effect of Bardeen-Herring correlation on vacancy diffusion in anisotropic crystals, Phys. Rev. 124 (1961) 1723-1730.

[38] P.B. Ghate. Screened interaction model for impurity diffusion in zinc, Phys. Rev. 133 (1964) 11671175 .

[39] A.D. Le Claire. Solute diffusion in dilute alloys, J. Nucl. Mater. 69 (1978) 70-96.

[40] B.C. Zhou, S.L. Shang, Y. Wang, Z.K. Liu. Data set for diffusion coefficients of alloying elements in dilute Mg alloys from first-principles, Data in Brief (2015) submitted.

[41] G. Kresse, D. Joubert. From ultrasoft pseudopotentials to the projector augmented-wave method, Phys. Rev. B 59 (1999) 1758-1775.

[42] G. Kresse, J. Furthmuller. Efficient iterative schemes for ab initio total-energy calculations using a plane-wave basis set, Phys. Rev. B 54 (1996) 11169-11186.

[43] P.E. Blochl, O. Jepsen, O.K. Andersen. Improved tetrahedron method for Brillouin-zone integrations, Phys. Rev. B 49 (1994) 16223-16233.

[44] D. Simonovic, M.H.F. Sluiter. Impurity diffusion activation energies in Al from first principles, Phys. Rev. B 79 (2009) 054304.

[45] L. Huber, I. Elfimov, J. Rottler, M. Militzer. Ab initio calculations of rare-earth diffusion in magnesium, Physical Review B 85 (2012) 144301.

[46] S.L. Shang, L.G. Hector, Y. Wang, Z.K. Liu. Anomalous energy pathway of vacancy migration and self-diffusion in hcp Ti, Phys. Rev. B 83 (2011) 224104.

[47] Y. Wang, Z.K. Liu, L.Q. Chen. Thermodynamic properties of Al, Ni, NiAl, and Ni3Al from firstprinciples calculations, Acta Mater. 52 (2004) 2665-2671.

[48] V.L. Moruzzi, J.F. Janak, K. Schwarz. Calculated thermal properties of metals, Phys. Rev. B 37 (1988) 790-799.

[49] X.L. Liu, B.K. VanLeeuwen, S.L. Shang, Y. Du, Z.K. Liu. On the scaling factor in Debye-Gruneisen model: A case study of the Mg-Zn binary system, Comput. Mater. Sci. 98 (2015) 34-41.

[50] S.L. Shang, A. Saengdeejing, Z.G. Mei, D.E. Kim, H. Zhang, S. Ganeshan, Y. Wang, Z.K. Liu. Firstprinciples calculations of pure elements: Equations of state and elastic stiffness constants, Comput. Mater. Sci. 48 (2010) 813-826. 
[51] S.L. Shang, Y. Wang, P. Guan, W.Y. Wang, H.Z. Fang, T. Anderson, Z.K. Liu. Insight into structural, elastic, phonon, and thermodynamic properties of $\alpha$-sulfur and energy-related sulfides: a comprehensive first-principles study, J. Mater. Chem. A 3 (2015) 8002-8014.

[52] C.J. Beevers. Electrical resistivity observations on quenched and cold-worked magnesium, Acta Metall. 11 (1963) 1029-1034.

[53] C. Mairy, J. Hillairet, D. Schumacher. Energie de formation et concentration d'équilibre des lacunes dans le magnésium, Acta Metall. 15 (1967) 1258-1261.

[54] C. Janot, D. Mallejac, B. George. Vacancy-formation energy and entropy in magnesium single crystals, Phys. Rev. B 2 (1970) 3088-3098.

[55] P. Tzanétakis, J. Hillairet, G. Revel. The formation energy of vacancies in aluminium and magnesium, Phys. Status Solidi B 75 (1976) 433-439.

[56] D. Segers, M. Dorikens, L. Dorikens-Vanpraet. Evidence for positron trapping in magnetism from dopplerbroadening measurements, Solid State Commun. 36 (1980) 943-945.

[57] P. Hautojärvi, J. Johansson, A. Vehanen, J. Ylikauppila, J. Hillairet, P. Tzanetakis. Trapping of positrons at vacancies in magnesium, App. Phys. A-Mater. 27 (1982) 49-56.

[58] P. Tzanétakis. PhD Thesis, University of Grenoble (1978).

[59] N. Chetty, M. Weinert, T.S. Rahman, J.W. Davenport. Vacancies and impurities in aluminum and magnesium, Phys. Rev. B 52 (1995) 6313-6326.

[60] H. Krimmel, M. Fahnle. Ab initio calculation of the formation and migration energies for monovacancies in Mg, Phys. Rev. B 62 (2000) 5489-5491.

[61] G.M. Hood. Comments on positron-annihilation and the vacancy properties of Mg, Phys. Rev. B 26 (1982) 1036-1037.

[62] R. Nazarov, T. Hickel, J. Neugebauer. Vacancy formation energies in fcc metals: Influence of exchange-correlation functionals and correction schemes, Phys. Rev. B 85 (2012) 144118.

[63] K. Carling, G. Wahnstrom, T.R. Mattsson, A.E. Mattsson, N. Sandberg, G. Grimvall. Vacancies in metals: From first-principles calculations to experimental data, Phys. Rev. Lett. 85 (2000) 3862-3865.

[64] S. Kurth, J.P. Perdew, P. Blaha. Molecular and solid-state tests of density functional approximations: LSD, GGAs, and meta-GGAs, Int. J. Quantum Chem 75 (1999) 889-909.

[65] A. Glensk, B. Grabowski, T. Hickel, J. Neugebauer. Breakdown of the Arrhenius law in describing vacancy formation energies: The importance of local anharmonicity revealed by Ab initio thermodynamics, Phys Rev X 4 (2014) 011018.

[66] H.R. Vydyanath, D.H. Sastry, K.I. Vasu. On the kinetics of clustering in a magnesium-3\% zinc alloy, Phys. Status Solidi B 29 (1968) K137-K140.

[67] A.R. Rao, C. Suryanarayana. Solute-vacancy binding energies in magnesium alloys, Phys. Status Solidi A 45 (1978) K131-K133.

[68] Y. Zhong, J.O. Sofo, A.A. Luo, Z.K. Liu. Thermodynamics modeling of the $\mathrm{Mg}$-Sr and $\mathrm{Ca}-\mathrm{Mg}-\mathrm{Sr}$ systems, J. Alloys Compd. 421 (2006) 172-178.

[69] P.G. Shewmon, F.N. Rhines. Rate of self-diffusion in polycrystalline magnesium, Trans. AIME 200 (1954) 1021-1025.

[70] P.G. Shewmon. Self-diffusion in magnesium single crystals, Trans. AIME 206 (1956) 918-922.

[71] J. Combronde, G. Brebec. Anisotropy for self diffusion in magnesium, Acta Metall. 19 (1971) 13931399.

[72] N.S. Kulkarni, R.J.B. Warmack, B. Radhakrishnan, J.L. Hunter, Y. Sohn, K.R. Coffey, G.E. Murch, I.V. Belova. Overview of SIMS-based experimental studies of tracer diffusion in solids and application to Mg self-diffusion, J. Phase Equilib. Diffus. 35 (2014) 762-778.

[73] C. Freysoldt, B. Grabowski, T. Hickel, J. Neugebauer, G. Kresse, A. Janotti, C.G. Van de Walle. First-principles calculations for point defects in solids, Rev. Mod. Phys. 86 (2014) 253-305.

[74] L.D. Calvert, P. Villars. Pearson's handbook of crystallographic data for intermetallic phases, 1991.

[75] A. van de Walle, G. Ceder. The effect of lattice vibrations on substitutional alloy thermodynamics, Rev. Mod. Phys. 74 (2002) 11-45.

[76] V.F. Yerko, V.F. Zelenskiy, V.S. Krasnorustskiy. Phys. Met. Metallogr. 22 (1966) 112. 
[77] K. Lal. Report No. CEA-R 3136 (Saclay) (1967) 3136.

[78] L.V. Pavlinov, A.M. Gladyshev, V.N. Bykov. Phys. Met. Metallogr. 26 (1968) 53.

[79] J. Combronde, G. Brebec. Diffusion of Ag, Cd, In, Sn and Sb in magnesium, Acta Metall. 20 (1972) $37-44$.

[80] S.I. Fujikawa. Impurity diffusion of manganese in magnesium, J. Japan Inst. Light Metals 42 (1992) 826-827.

[81] I. Stloukal, J. Čermák. Grain boundary diffusion of $67 \mathrm{Ga}$ in polycrystalline magnesium, Scripta Mater. 49 (2003) 557-562.

[82] J. Čermák, I. Stloukal. Diffusion of $65 \mathrm{Zn}$ in $\mathrm{Mg}$ and in Mg-xAl solid solutions, Phys. Status Solidi A 203 (2006) 2386-2392.

[83] S. Brennan, K. Bermudez, N.S. Kulkarni, Y. Sohn. Interdiffusion in the Mg-Al System and Intrinsic Diffusion in $\beta-M g 2 A 13$, Metall. Mater. Trans. A 43A (2012) 4043-4052.

[84] S. Brennan, A.P. Warren, K.R. Coffey, N. Kulkarni, P. Todd, M. Kilmov, Y. Sohn. Aluminum impurity diffusion in magnesium, J. Phase Equilib. Diffus. 33 (2012) 121-125.

[85] S.K. Das, Y.M. Kim, T.K. Ha, R. Gauvin, I.H. Jung. Anisotropic diffusion behavior of Al in Mg: diffusion couple study using Mg single crystal, Metall. Mater. Trans. A 44A (2013) 2539-2547.

[86] S.K. Das, Y.M. Kim, T.K. Ha, I.H. Jung. Investigation of anisotropic diffusion behavior of Zn in hcp $\mathrm{Mg}$ and interdiffusion coefficients of intermediate phases in the Mg-Zn system, CALPHAD 42 (2013) $51-58$.

[87] S.K. Das, Y.B. Kang, T. Ha, I.H. Jung. Thermodynamic modeling and diffusion kinetic experiments of binary Mg-Gd and Mg-Y systems, Acta Mater. 71 (2014) 164-175.

[88] C.C. Kammerer, N.S. Kulkarni, R.J. Warmack, Y.H. Sohn. Interdiffusion and impurity diffusion in polycrystalline Mg solid solution with Al or Zn, J. Alloys Compd. 617 (2014) 968-974.

[89] Z.L. Bryan, P. Alieninov, I.S. Berglund, M.V. Manuel. A diffusion mobility database for magnesium alloy development, CALPHAD 48 (2015) 123-130.

[90] A.A. Nayeb-Hashemi, J.B. Clark. Binary Alloy Phase Diagrams, Second Edition, T.B. Massalski (editor-in-chief), ASM International, 1990. 


\section{Tables and table captions}

Table 1

Comparison of experimental and first-principles calculated vacancy formation energies $\Delta E_{f}^{0}$ and equilibrium lattice parameters $a_{0}$ and $c_{0}$ in hcp Mg. First-principles results are calculated using different $\mathrm{X}-\mathrm{C}$ functionals of LDA, GGA, and PBEsol at $0 \mathrm{~K}$ and with various supercell sizes. Note that the experimentally measured vacancy formation energies are usually assumed to be constant with respect to temperature.

\begin{tabular}{|c|c|c|c|c|c|}
\hline Method & Supercell size (sites) & $a_{0}(\AA)$ & $c_{0}(\AA)$ & Vacancy formation energy $(\mathrm{eV})$ & Reference \\
\hline LDA & 96 & 3.112 & 5.041 & 0.83 & Chetty et al. [59] \\
\hline LDA & 54 & 3.12 & 5.06 & $0.83 \pm 0.07$ & Krimmel and Fahnle [60] \\
\hline LDA & 36 & 3.141 & 5.065 & 0.84 & Mantina et al. [14] \\
\hline LDA & 36 & 3.133 & 5.079 & 0.81 & Ganeshan et al. [25] \\
\hline LDA & $48,64,96,144$ & & & $0.83 \pm 0.01$ & Shin and Wolverton [30] \\
\hline GGA & 36 & 3.195 & 5.176 & 0.72 & Ganeshan et al. [25] \\
\hline GGA & $48,64,96,144$ & 3.197 & 5.173 & $0.74 \pm 0.05$ & Shin and Wolverton [30] \\
\hline PBEsol & 36 & 3.172 & 5.152 & 0.83 & This work \\
\hline PBEsol & 54 & 3.176 & 5.149 & 0.85 & This work \\
\hline PBEsol & 64 & $3.175^{a}$ & $5.154^{a}$ & 0.86 & This work \\
\hline PBEsol & 96 & 3.165 & 5.190 & 0.85 & This work \\
\hline Expt. $\Delta \rho_{q}^{b}$ & & & & $0.89 \pm 0.06$ & Beevers [52] \\
\hline Expt. $\Delta \rho_{q}$ & & & & $0.79 \pm 0.03$ & Tzanétakis et al. [55] \\
\hline Expt. $\Delta \rho_{q}$ & & & & $0.83 \pm 0.03$ & Tzanétakis [58] \\
\hline Expt. $\Delta \rho_{e q}{ }^{c}$ & & & & $0.81 \pm 0.02$ & Mairy et al. [53] \\
\hline Expt. DD ${ }^{d}$ & & & & $0.58 \pm 0.01$ & Janot et al. [54] \\
\hline Expt. PAS ${ }^{e}$ & & & & $0.85_{-0.05}^{+0.07}$ & Segers et al. [56] \\
\hline Expt. PAS & & & & 0.85 & Hautojärvi et al. [57] \\
\hline
\end{tabular}

${ }^{a}$ Experimental lattice parameters of $\mathrm{Mg} a_{0}=3.209 \AA$ and $c_{0}=5.211 \AA$ at room temperature [74]

${ }^{b} \Delta \rho_{q}$ : electrical resistivity of quenched samples

${ }^{c} \Delta \rho_{e q}:$ electrical resistivity of thermal equilibrium samples

${ }^{d}$ DD: differential dilatometry

${ }^{e}$ PAS: positron annihilation spectroscopy 
Table 2

First-principles predicted properties of solutes in hep $\mathrm{Mg}$ by the $\mathrm{X}-\mathrm{C}$ functional of PBEsol, including the volume difference, bulk modulus, solute-vacancy binding energies and migration barriers. Here, $\Delta V_{X}$ indicates the volume difference induced by placing a single solute into pure $\mathrm{Mg}$, see Eq. (19). $B$ is the bulk modulus of $\mathrm{Mg}_{63} \mathrm{X}\left(\mathrm{Mg}_{95} \mathrm{X}\right.$ for $\mathrm{Ba}, \mathrm{Bi}, \mathrm{Ca}, \mathrm{K}, \mathrm{Pb}, \mathrm{Sr}$, and $\left.\mathrm{Y}\right) . E_{\text {bind }}^{\text {basal }}$ and $E_{\text {bind }}^{\text {normal }}$ are the solutevacancy binding energies of solute and vacancy on the same basal plane and between adjacent basal planes of hcp $\mathrm{Mg}$, respectively. $E_{X}$ and $E_{X}^{\prime}$ are the solute migration barriers for solute-vacancy exchange within the basal plane and between adjacent basal planes, respectively. $E_{\text {mix }}$ is the dilute mixing energy given in units of $\mathrm{eV}$ per atom of solute. $S$ is the maximum solid solubility of each element in $\mathrm{Mg}$ from experiments [90].

\begin{tabular}{|c|c|c|c|c|c|c|c|c|}
\hline Solute & $\Delta V_{X}\left(\AA^{3}\right)$ & $B(\mathrm{GPa})$ & $E_{\text {bind }}^{\text {basal }}(\mathrm{eV})$ & $E_{\text {bind }}^{\text {normal }}(\mathrm{eV})$ & $E_{X}(\mathrm{eV})$ & $E_{X}^{\prime}(\mathrm{eV})$ & $E_{m i x}(\mathrm{eV} / \mathrm{sol})$ & $S$ (at.\%) \\
\hline $\mathrm{Mg}$ & 0 & 37.9 & - & - & 0.41 & 0.44 & - & - \\
\hline $\mathrm{Ag}$ & -13.3 & 38.6 & 0.05 & 0.04 & 0.63 & 0.68 & -0.33 & 2.58 \\
\hline $\mathrm{Al}$ & -7.8 & 38.3 & $0.02^{a}$ & 0.02 & 0.46 & 0.48 & 0.12 & 11.8 \\
\hline As & -9.4 & 38.5 & 0.15 & 0.15 & 0.44 & 0.45 & -0.49 & $\sim 0$ \\
\hline $\mathrm{Au}$ & -17.8 & 39.0 & 0.12 & 0.08 & 0.77 & 0.82 & -1.12 & 0.1 \\
\hline $\mathrm{Ba}$ & 34.0 & 36.8 & 0.67 & 0.58 & 0 & 0 & 0.76 & 0.002 \\
\hline $\mathrm{Be}$ & -16.3 & 38.4 & 0.09 & 0.06 & 0.57 & 0.58 & 1.31 & $\mathrm{~N} / \mathrm{A}^{c}$ \\
\hline $\mathrm{Bi}$ & 6.9 & 38.2 & 0.11 & 0.12 & 0.44 & 0.44 & -0.39 & 1.12 \\
\hline $\mathrm{Ca}$ & 15.1 & 37.3 & 0.08 & 0.10 & 0.14 & 0.18 & 0.03 & 0.44 \\
\hline $\mathrm{Cd}$ & -5.0 & 38.3 & 0.05 & 0.04 & 0.46 & 0.49 & -0.28 & 100 \\
\hline Co & -26.1 & 38.6 & 0.17 & 0.07 & 1.27 & 1.34 & 1.04 & $\sim 0$ \\
\hline $\mathrm{Cr}$ & -12.0 & 38.4 & -0.08 & -0.09 & 0.81 & 0.84 & 1.34 & N/A \\
\hline $\mathrm{Cu}$ & -18.0 & 38.6 & 0.10 & 0.06 & 0.65 & 0.67 & 0.35 & 0.234 \\
\hline $\mathrm{Fe}$ & -20.4 & 38.5 & 0.03 & -0.02 & 0.96 & 1.02 & 1.30 & 0.00043 \\
\hline $\mathrm{Ga}$ & -9.1 & 38.2 & 0.06 & 0.05 & 0.49 & 0.52 & -0.23 & 3.14 \\
\hline Ge & -9.6 & 38.4 & 0.09 & 0.08 & 0.56 & 0.61 & -0.20 & 0.003 \\
\hline $\mathrm{Hf}$ & -3.3 & 38.7 & -0.26 & -0.26 & 0.82 & 0.92 & 0.76 & $\sim 0$ \\
\hline $\mathrm{Hg}$ & -7.3 & 38.4 & 0.07 & 0.06 & 0.45 & 0.48 & -0.68 & 1.2 \\
\hline In & -1.4 & 38.2 & 0.06 & 0.06 & 0.47 & 0.49 & -0.35 & 19.4 \\
\hline Ir & -30.0 & 39.7 & 0.19 & 0.10 & 1.67 & 1.63 & -0.59 & $\sim 0$ \\
\hline K & 25.5 & 36.7 & 0.40 & 0.37 & 0 & 0 & 1.28 & $\sim 0$ \\
\hline $\mathrm{Li}$ & -4.4 & 37.5 & 0.003 & $\sim 0$ & 0.36 & 0.39 & -0.16 & 17 \\
\hline $\mathrm{Mn}$ & -14.0 & 38.3 & -0.06 & -0.07 & 0.74 & 0.81 & 0.96 & 0.996 \\
\hline Mo & -19.4 & 39.2 & -0.18 & -0.21 & 1.54 & 1.68 & 1.61 & $\sim 0$ \\
\hline $\mathrm{Na}$ & 7.1 & 37.0 & 0.08 & 0.08 & 0.18 & 0.19 & 0.38 & 0.5 \\
\hline $\mathrm{Nb}$ & -11.9 & 39.0 & -0.28 & -0.29 & 1.19 & 1.33 & 1.23 & $\sim 0$ \\
\hline $\mathrm{Ni}$ & -24.3 & 38.8 & 0.17 & 0.08 & 1.03 & 1.07 & 0.35 & $<0.04$ \\
\hline Os & -29.6 & 39.7 & 0.10 & 0.01 & 1.91 & 1.80 & 0.88 & N/A \\
\hline $\mathrm{Pb}$ & 4.4 & 37.9 & 0.08 & 0.09 & 0.44 & 0.45 & -0.26 & 7.75 \\
\hline $\mathrm{Pd}$ & -22.4 & 39.1 & 0.13 & 0.07 & 1.04 & 1.11 & -1.17 & $<0.23$ \\
\hline $\mathrm{Pt}$ & -26.3 & 39.4 & 0.20 & 0.12 & 1.26 & 1.31 & -1.54 & $\sim 0$ \\
\hline $\mathrm{Re}$ & -26.1 & 39.6 & -0.05 & -0.11 & 1.91 & 1.86 & 1.69 & N/A \\
\hline $\mathrm{Rh}$ & -27.3 & 39.4 & 0.15 & 0.07 & 1.44 & 1.49 & -0.77 & N/A \\
\hline $\mathrm{Ru}$ & -27.9 & 39.5 & 0.09 & 0.01 & 1.68 & 1.70 & 0.35 & N/A \\
\hline $\mathrm{Sb}$ & 1.2 & 38.3 & 0.12 & 0.13 & 0.45 & 0.48 & -0.51 & $<0.04$ \\
\hline $\mathrm{Sc}$ & 1.2 & 38.2 & -0.16 & -0.14 & 0.50 & 0.58 & -0.05 & 29 \\
\hline $\mathrm{Se}$ & -4.6 & 38.2 & 0.26 & 0.26 & 0.08 & 0.07 & -0.86 & N/A \\
\hline $\mathrm{Si}$ & -12.7 & 38.6 & 0.09 & 0.08 & 0.61 & 0.66 & 0.29 & 0.005 \\
\hline $\mathrm{Sn}$ & -0.2 & 38.2 & 0.08 & 0.08 & 0.52 & 0.55 & -0.46 & 3.35 \\
\hline $\mathrm{Sr}$ & 22.5 & 37.1 & 0.30 & 0.29 & 0.01 & 0.02 & 0.44 & 0.03 \\
\hline $\mathrm{Ta}$ & -12.4 & 39.1 & -0.29 & -0.30 & 1.27 & 1.41 & 1.67 & N/A \\
\hline $\mathrm{Tc}$ & -24.4 & 39.4 & -0.04 & -0.10 & 1.71 & 1.78 & 1.07 & N/A \\
\hline
\end{tabular}




\begin{tabular}{lrrrrrrrl}
\hline $\mathrm{Te}$ & 5.3 & 38.0 & 0.20 & 0.22 & 0.18 & 0.19 & -0.57 & N/A \\
$\mathrm{Ti}$ & -8.9 & 38.7 & -0.21 & -0.22 & 0.84 & 0.95 & 0.79 & 0.12 \\
$\mathrm{Tl}$ & 1.6 & 37.9 & 0.04 & 0.04 & 0.39 & 0.40 & -0.29 & 15.4 \\
$\mathrm{~V}$ & -11.8 & 38.6 & -0.14 & -0.15 & 0.90 & 1.00 & 1.32 & $\sim 0$ \\
$\mathrm{~W}$ & -19.7 & 39.3 & -0.20 & -0.23 & 1.68 & 1.77 & 2.29 & $\mathrm{~N} / \mathrm{A}$ \\
$\mathrm{Y}$ & 11.5 & 37.9 & -0.07 & -0.06 & 0.28 & 0.33 & -0.06 & 4.7 \\
$\mathrm{Zn}$ & -11.1 & 38.2 & $0.05^{b}$ & 0.04 & 0.46 & 0.48 & 0.05 & 2.5 \\
$\mathrm{Zr}$ & -2.1 & 38.6 & -0.25 & -0.24 & 0.76 & 0.86 & 0.37 & 1.042 \\
\hline
\end{tabular}

${ }^{a}$ Experimental $E_{\text {bind }}(A l-V a)=0.29 \pm 0.02 \mathrm{eV}$ [67]

${ }^{b}$ Experimental $E_{\text {bind }}(Z n-V a)=0.07 \pm 0.02 \mathrm{eV}$ [66]

${ }^{c} \mathrm{~N} / \mathrm{A}$ indicates that experiments of solid solubility in $\mathrm{Mg}$ are not available in the literature. 
Table 3

Energy barriers $(\mathrm{eV})$ of vacancy migration for various solutes in hcp $\mathrm{Mg}$. The subscripts refer to the migration pathways indicated in Figure 3.

\begin{tabular}{lcccccccc}
\hline Solute & $E_{X}$ & $E_{X}^{\prime}$ & $E_{a}$ & $E_{a}^{\prime}$ & $E_{b}^{\prime}$ & $E_{b}$ & $E_{c}$ & $E_{c}^{\prime}$ \\
\hline $\mathrm{Zn}$ & 0.46 & 0.48 & 0.27 & 0.26 & 0.27 & 0.38 & 0.44 & 0.42 \\
$\mathrm{~Pb}$ & 0.45 & 0.46 & 0.40 & 0.45 & 0.41 & 0.42 & 0.45 & 0.43 \\
$\mathrm{Bi}$ & 0.44 & 0.44 & 0.47 & 0.48 & 0.43 & 0.43 & 0.45 & 0.43 \\
$\mathrm{Ca}$ & 0.14 & 0.18 & 0.73 & 0.75 & 0.70 & 0.55 & 0.37 & 0.37 \\
$\mathrm{Na}$ & 0.18 & 0.19 & 0.55 & 0.56 & 0.52 & 0.48 & 0.40 & 0.40 \\
$\mathrm{Se}$ & 0.08 & 0.07 & 0.34 & 0.35 & 0.38 & 0.45 & 0.47 & 0.42 \\
$\mathrm{Sr}$ & 0.01 & 0.02 & 0.96 & 0.95 & 0.90 & 0.76 & 0.47 & 0.43 \\
$\mathrm{Te}$ & 0.18 & 0.19 & 0.48 & 0.50 & 0.44 & 0.45 & 0.49 & 0.46 \\
$\mathrm{Y}$ & 0.28 & 0.33 & 0.67 & 0.68 & 0.63 & 0.47 & 0.33 & 0.34 \\
\hline
\end{tabular}


Table 4

Predicted dilute solute (impurity) diffusion coefficients by the X-C functional of PBEsol compared with available experimental values. $D_{0 \perp}$ and $D_{0 \square}$ are the diffusion pre-factors $\left(\mathrm{m}^{2} / \mathrm{s}\right)$ for the diffusion components perpendicular and parallel to the $c$ axis, respectively. $Q_{\perp}$ and $Q_{\square}$ are the diffusion activation energies $(\mathrm{kJ} / \mathrm{mol})$ for the diffusion components perpendicular and parallel to the $c$ axis, respectively. Trange is the temperature range in which the experimental measurements were performed. If only one set of average $D_{0}$ and $Q$ data is listed for a solute, it indicates that the data was measured from polycrystalline $\mathrm{Mg}$ sample without anisotropy.

\begin{tabular}{|c|c|c|c|c|c|c|c|c|c|c|}
\hline \multirow{2}{*}{ Solute } & \multicolumn{4}{|c|}{ Calculation } & \multicolumn{5}{|l|}{ Experiment } & \multirow{2}{*}{ Ref. } \\
\hline & $D_{0 \perp}$ & $Q_{\perp}$ & $D_{0 \square}$ & $Q_{\square}$ & $D_{0 \perp}$ & $Q_{\perp}$ & $D_{0 \square}$ & $Q$ & T-range (K) & \\
\hline \multirow[t]{4}{*}{$\mathrm{Mg}$} & $6.83 \times 10^{-5}$ & 129.4 & $5.90 \times 10^{-5}$ & 130.7 & $1.00 \times 10^{-4}$ & 134.0 & & & $741 \sim 900$ & [69] \\
\hline & & & & & $1.50 \times 10^{-4}$ & 136.1 & $1.00 \times 10^{-4}$ & 134.8 & 741 908 & [70] \\
\hline & & & & & $1.75 \times 10^{-4}$ & 138.0 & $1.78 \times 10^{-4}$ & 139.0 & 775 906 & [71] \\
\hline & & & & & $1.32 \times 10^{-5}$ & 121.3 & $3.30 \times 10^{-6}$ & 114.6 & $523 \sim 900$ & [72] \\
\hline \multirow[t]{2}{*}{$\mathrm{Ag}^{*}$} & $7.54 \times 10^{-5}$ & 145.2 & $1.26 \times 10^{-4}$ & 151.9 & $3.40 \times 10^{-5}$ & 119.3 & & & $750 \sim 894$ & [77] \\
\hline & & & & & $1.79 \times 10^{-3}$ & 148.1 & $3.62 \times 10^{-4}$ & 133.1 & $752 \sim 912$ & [79] \\
\hline \multirow[t]{4}{*}{$\mathrm{Al}^{*}$} & $3.44 \times 10^{-5}$ & 129.3 & $3.11 \times 10^{-5}$ & 131.5 & $3.90 \times 10^{-3}$ & 155.0 & & & $573 \sim 673$ & [84] \\
\hline & & & & & $4.20 \times 10^{-7}$ & 114.7 & & & $573 \sim 673$ & [83] \\
\hline & & & & & $6.25 \times 10^{-5}$ & 139.3 & & & $623 \sim 723$ & [88] \\
\hline & & & & & $4.86 \times 10^{-3}$ & 154.5 & $9.51 \times 10^{-3}$ & 159.5 & $638 \sim 693$ & [85] \\
\hline As & $4.36 \times 10^{-5}$ & 116.8 & $3.45 \times 10^{-5}$ & 117.5 & & & & & & \\
\hline $\mathrm{Au}$ & $5.91 \times 10^{-5}$ & 151.5 & $7.77 \times 10^{-5}$ & 160.0 & & & & & & \\
\hline $\mathrm{Be}$ & $3.16 \times 10^{-5}$ & 133.2 & $3.45 \times 10^{-5}$ & 136.6 & $5.60 \times 10^{-4}$ & 156.9 & & & $773 \sim 873$ & [76] \\
\hline $\mathrm{Bi}$ & $1.66 \times 10^{-6}$ & 111.7 & $1.37 \times 10^{-6}$ & 110.5 & & & & & & \\
\hline $\mathrm{Ca}^{*}$ & $4.61 \times 10^{-4}$ & 119.1 & $3.41 \times 10^{-4}$ & 116.9 & & & & & & \\
\hline $\mathrm{Cd}$ & $6.72 \times 10^{-5}$ & 129.0 & $1.15 \times 10^{-4}$ & 132.8 & $4.60 \times 10^{-5}$ & 132.6 & $1.29 \times 10^{-4}$ & 140.6 & $733 \sim 899$ & [79] \\
\hline Co & $3.69 \times 10^{-5}$ & 197.8 & $2.10 \times 10^{-5}$ & 210.9 & & & & & & \\
\hline $\mathrm{Cr}$ & $4.13 \times 10^{-5}$ & 169.4 & $8.16 \times 10^{-5}$ & 177.8 & & & & & & \\
\hline $\mathrm{Cu}$ & $1.49 \times 10^{-5}$ & 138.6 & $3.18 \times 10^{-5}$ & 145.8 & & & & & & \\
\hline $\mathrm{Fe}$ & $8.86 \times 10^{-5}$ & 180.6 & $2.61 \times 10^{-4}$ & 192.6 & $4.00 \times 10^{-10}$ & 88.8 & & & $673 \sim 873$ & [78] \\
\hline $\mathrm{Ga}$ & $7.82 \times 10^{-3}$ & 144.0 & $7.67 \times 10^{-3}$ & 147.6 & $1.20 \times 10^{-4}$ & 134.3 & & & $639 \sim 872$ & [81] \\
\hline $\mathrm{Ge}$ & $3.45 \times 10^{-5}$ & 134.7 & $3.06 \times 10^{-5}$ & 138.4 & & & & & & \\
\hline $\mathrm{Hf}$ & $1.86 \times 10^{-5}$ & 192.5 & $1.84 \times 10^{-5}$ & 201.1 & & & & & & \\
\hline $\mathrm{Hg}$ & $8.27 \times 10^{-5}$ & 125.8 & $3.78 \times 10^{-5}$ & 126.6 & & & & & & \\
\hline \multirow[t]{2}{*}{ In } & $5.97 \times 10^{-5}$ & 127.9 & $5.47 \times 10^{-5}$ & 130.2 & $1.88 \times 10^{-4}$ & 142.3 & $1.75 \times 10^{-4}$ & 143.3 & 748 906 & [79] \\
\hline & & & & & $5.20 \times 10^{-6}$ & 118.9 & & & $745 \sim 883$ & [77] \\
\hline Ir & $5.57 \times 10^{-6}$ & 224.5 & $5.74 \times 10^{-6}$ & 231.8 & & & & & & \\
\hline $\mathrm{Li}^{*}$ & $5.08 \times 10^{-5}$ & 124.2 & $6.34 \times 10^{-5}$ & 127.2 & & & & & & \\
\hline $\mathrm{Mn}^{*}$ & $2.49 \times 10^{-4}$ & 170.6 & $3.32 \times 10^{-4}$ & 178.0 & $7.60 \times 10^{-5}$ & 154.0 & & & 843,903 & [80] \\
\hline Mo & $3.30 \times 10^{-6}$ & 250.8 & $5.01 \times 10^{-6}$ & 266.4 & & & & & & \\
\hline $\mathrm{Na}$ & $1.44 \times 10^{-4}$ & 120.8 & $1.31 \times 10^{-4}$ & 120.3 & & & & & & \\
\hline $\mathrm{Nb}$ & $3.62 \times 10^{-5}$ & 232.8 & $4.97 \times 10^{-5}$ & 246.5 & & & & & & \\
\hline $\mathrm{Ni}$ & $2.21 \times 10^{-4}$ & 175.8 & $2.30 \times 10^{-4}$ & 185.4 & $1.20 \times 10^{-9}$ & 95.9 & & & $673 \sim 873$ & {$[78]$} \\
\hline Os & $2.25 \times 10^{-3}$ & 266.9 & $4.91 \times 10^{-3}$ & 274.5 & & & & & & \\
\hline $\mathrm{Pb}$ & $6.53 \times 10^{-6}$ & 119.4 & $4.46 \times 10^{-6}$ & 119.2 & & & & & & \\
\hline $\mathrm{Pd}$ & $3.93 \times 10^{-5}$ & 177.0 & $4.76 \times 10^{-5}$ & 188.4 & & & & & & \\
\hline $\mathrm{Pt}$ & $2.41 \times 10^{-5}$ & 190.5 & $3.41 \times 10^{-5}$ & 203.2 & & & & & & \\
\hline $\mathrm{Re}$ & $7.73 \times 10^{-6}$ & 273.0 & $8.29 \times 10^{-6}$ & 276.4 & & & & & & \\
\hline $\mathrm{Rh}$ & $3.06 \times 10^{-5}$ & 212.4 & $2.34 \times 10^{-5}$ & 223.6 & & & & & & \\
\hline $\mathrm{Ru}$ & $8.91 \times 10^{-3}$ & 257.2 & $9.56 \times 10^{-3}$ & 266.2 & & & & & & \\
\hline $\mathrm{Sb}$ & $1.89 \times 10^{-5}$ & 118.4 & $2.09 \times 10^{-5}$ & 120.3 & $3.27 \times 10^{-4}$ & 138.1 & $2.57 \times 10^{-4}$ & 137.2 & $781 \sim 896$ & [79] \\
\hline $\mathrm{Sc}$ & $3.45 \times 10^{-5}$ & 152.2 & $2.52 \times 10^{-5}$ & 156.1 & & & & & & \\
\hline $\mathrm{Se}$ & $1.05 \times 10^{-4}$ & 100.2 & $6.90 \times 10^{-5}$ & 96.6 & & & & & & \\
\hline
\end{tabular}




\begin{tabular}{|c|c|c|c|c|c|c|c|c|c|c|}
\hline $\mathrm{Si}^{*}$ & $4.98 \times 10^{-5}$ & 139.3 & $5.37 \times 10^{-5}$ & 144.1 & & & & & & \\
\hline $\mathrm{Sn}^{*}$ & $4.61 \times 10^{-5}$ & 131.8 & $4.74 \times 10^{-5}$ & 134.2 & $1.84 \times 10^{-4}$ & 144.5 & $3.63 \times 10^{-4}$ & 149.9 & 748 902 & [79] \\
\hline $\mathrm{Sr}^{*}$ & $1.24 \times 10^{-5}$ & 99.3 & $1.73 \times 10^{-5}$ & 98.9 & & & & & & \\
\hline Ta & $3.78 \times 10^{-6}$ & 236.4 & $5.93 \times 10^{-6}$ & 254.0 & & & & & & \\
\hline $\mathrm{Tc}$ & $1.20 \times 10^{-4}$ & 262.7 & $1.69 \times 10^{-4}$ & 274.3 & & & & & & \\
\hline $\mathrm{Te}$ & $9.29 \times 10^{-5}$ & 113.2 & $8.73 \times 10^{-5}$ & 110.8 & & & & & & \\
\hline $\mathrm{Ti}$ & $1.54 \times 10^{-5}$ & 188.9 & $3.57 \times 10^{-5}$ & 200.8 & & & & & & \\
\hline $\mathrm{Tl}$ & $4.02 \times 10^{-5}$ & 121.3 & $4.44 \times 10^{-5}$ & 122.6 & & & & & & \\
\hline V & $5.09 \times 10^{-5}$ & 188.4 & $1.41 \times 10^{-4}$ & 200.4 & & & & & & \\
\hline W & $5.42 \times 10^{-6}$ & 268.6 & $6.07 \times 10^{-6}$ & 278.3 & & & & & & \\
\hline $\mathrm{Y}^{*}$ & $3.68 \times 10^{-6}$ & 119.2 & $5.04 \times 10^{-6}$ & 122.4 & $2.79 \times 10^{-8}$ & 97.9 & $3.21 \times 10^{-8}$ & 99.1 & $703 \sim 803$ & [87] \\
\hline \multirow[t]{4}{*}{$\mathrm{Zn} *$} & $1.59 \times 10^{-4}$ & 129.9 & $1.29 \times 10^{-4}$ & 132.4 & $4.10 \times 10^{-5}$ & 119.8 & & & $740 \sim 893$ & {$[77]$} \\
\hline & & & & & $1.05 \times 10^{-4}$ & 125.8 & & & $648 \sim 848$ & [82] \\
\hline & & & & & $2.90 \times 10^{-5}$ & 118.6 & & & $623 \sim 723$ & [88] \\
\hline & & & & & $4.98 \times 10^{-5}$ & 132.7 & $7.33 \times 10^{-5}$ & 135.5 & $553 \sim 603$ & [86] \\
\hline $\mathrm{Zr}^{*}$ & $1.42 \times 10^{-5}$ & 186.2 & $2.83 \times 10^{-5}$ & 195.3 & & & & & & \\
\hline
\end{tabular}

* Alloying elements commonly used in commercial Mg alloys. 


\section{Figures and figure captions}

\begin{tabular}{|c|c|c|c|c|c|c|c|c|c|c|c|c|c|c|c|}
\hline \multirow{2}{*}{\begin{tabular}{|l|} 
Li_sv \\
$\mathrm{Na}$ _pv
\end{tabular}} & \multirow{2}{*}{$\begin{array}{l}\mathrm{Be} \\
\mathrm{Mg}\end{array}$} & & & \multicolumn{4}{|c|}{ Mg polycrystalline sample } & & \multicolumn{3}{|c|}{ No expt. } & & & & \\
\hline & & & & $M g \sin$ & le crys & tal sam & & & & & & $\mathrm{Al}$ & $\mathrm{Si}$ & & \\
\hline K_sv & Ca_sv & Sc_sv & Ti_sv & V_sv & Cr_pv & Mn_pv & $\mathrm{Fe}$ & Co & $\mathrm{Ni}$ & $\mathrm{Cu}$ & $\mathrm{Zn}$ & Ga_d & Ge_d & As & $\mathrm{Se}$ \\
\hline & Sr_sv & Y_sv & Zr_sv & $\mathrm{Nb} \_s v$ & Mo_sv & Tc_pv & Ru_pv & Rh_pv & $\mathrm{Pd}$ & $\mathrm{Ag}$ & $\mathrm{Cd}$ & In_d & Sn_d & $\mathrm{Sb}$ & $\mathrm{Te}$ \\
\hline & Ba_sv & & Hf_pv & Ta_pv & W_pv & $\operatorname{Re}$ & Os & Ir & $\mathrm{Pt}$ & $\mathrm{Au}$ & $\mathrm{Hg}$ & TI_d & Pb_d & Bi_d & \\
\hline
\end{tabular}

Figure 1. 49 alloying elements in dilute hcp $\mathrm{Mg}$ studied in the present work together with available experiments of diffusion data (see Table 4 for details) denoted in the periodic table. The elemental names indicate the recommended standard potentials supplied by VASP used in the present work for each element. The extensions sv, pv, and $d$ mean the semi-core $s, p$, and $d$ states are treated as valence states as well, respectively. Note that the diffusion coefficients of $\mathrm{Ba}$ and $\mathrm{K}$ were not calculated because their direct migration barriers are vanishingly small. 


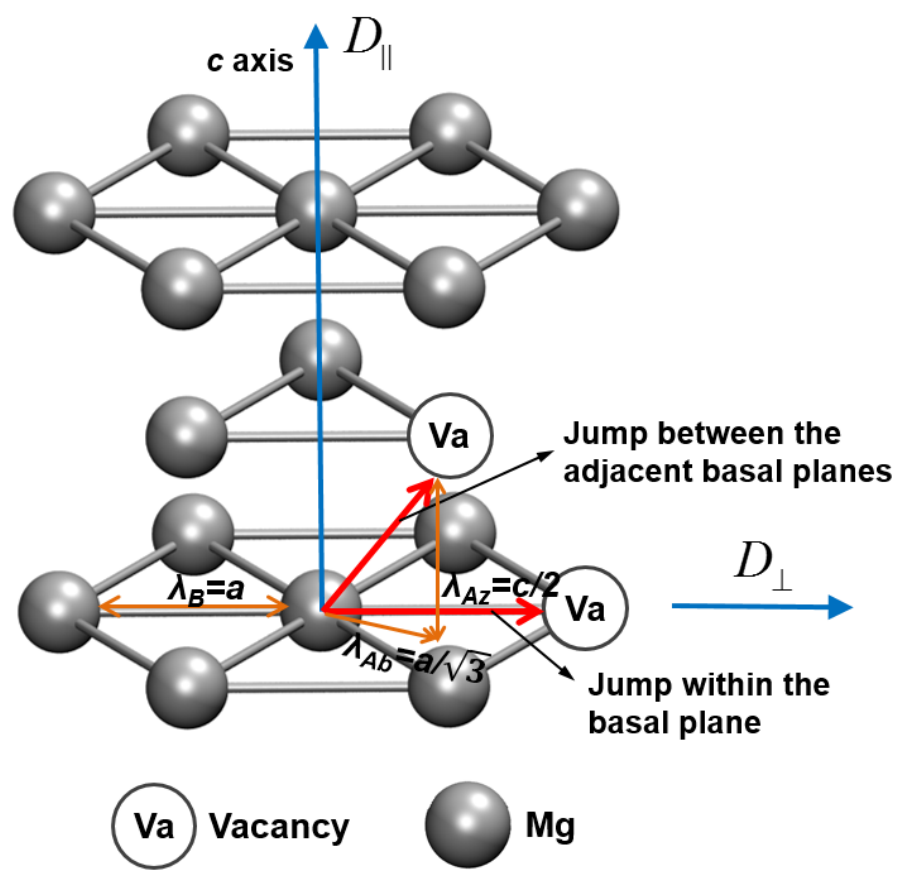

Figure 2. Illustration of vacancy-mediated diffusion jump components in an hcp lattice showing different jump distances $\left(\lambda_{B}, \lambda_{A b}\right.$, and $\left.\lambda_{A z}\right)$. The atom in the middle can exchange position with vacancies (red arrows) either within the same basal plane at a distance of $\lambda_{B}=a$, or between adjacent basal planes at distances of $\lambda_{A b}=a / \sqrt{3}$ along the basal plane and $\lambda_{A z}=c / 2$ along the $c$-axis. The jump components along basal plane with jump distances $\lambda_{B}$ and $\lambda_{A b}$ contribute to the diffusion coefficient $D_{\perp}(\perp c$-axis), while the jump component along distance $\lambda_{A z}$ contributes to the diffusion coefficient $D_{\square}(\| c$-axis). 

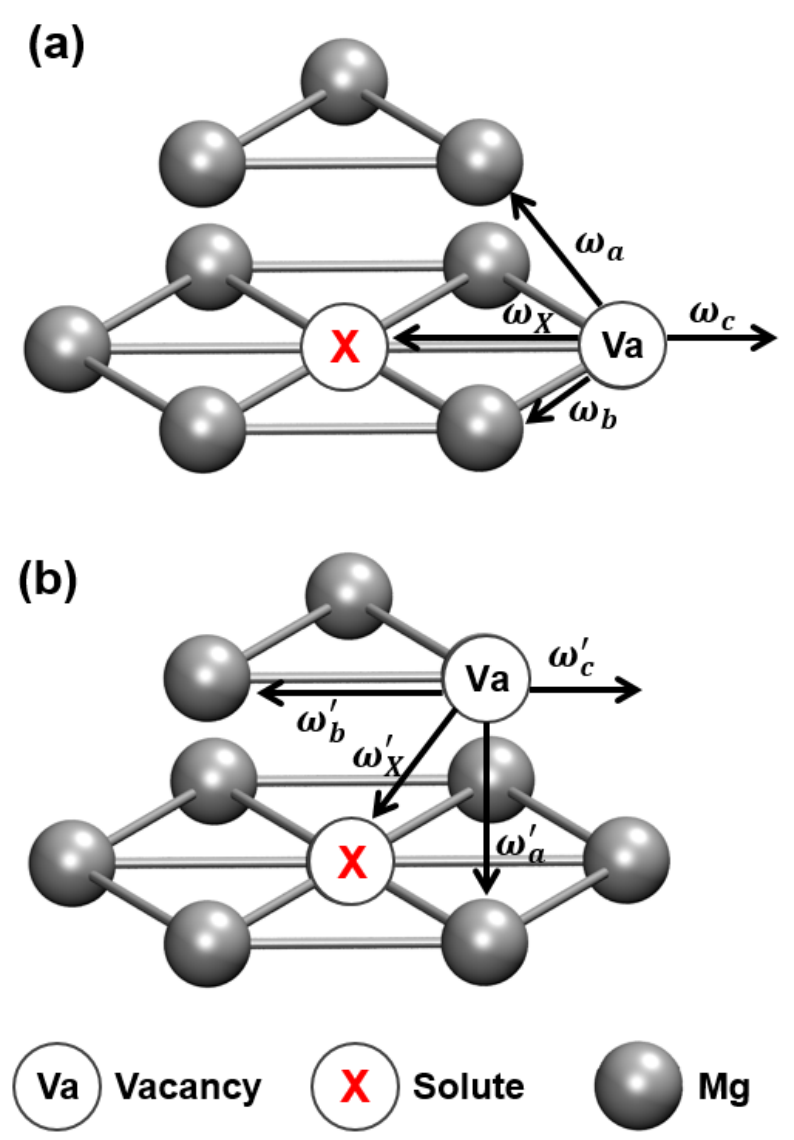

Figure 3. Illustration of the eight possible vacancy exchanges in an hop lattice for vacancy and solute starting (a) within the basal plane and (b) between adjacent basal planes. $\omega_{X}, \omega_{X}^{\prime}$ are the jump frequencies for the solutes $(\mathrm{X})$ and $\omega_{a}, \omega_{b}, \omega_{c}, \omega_{a}^{\prime}, \omega_{b}^{\prime}, \omega_{c}^{\prime}$ are the jump frequencies for the solvents $(\mathrm{Mg})$. 


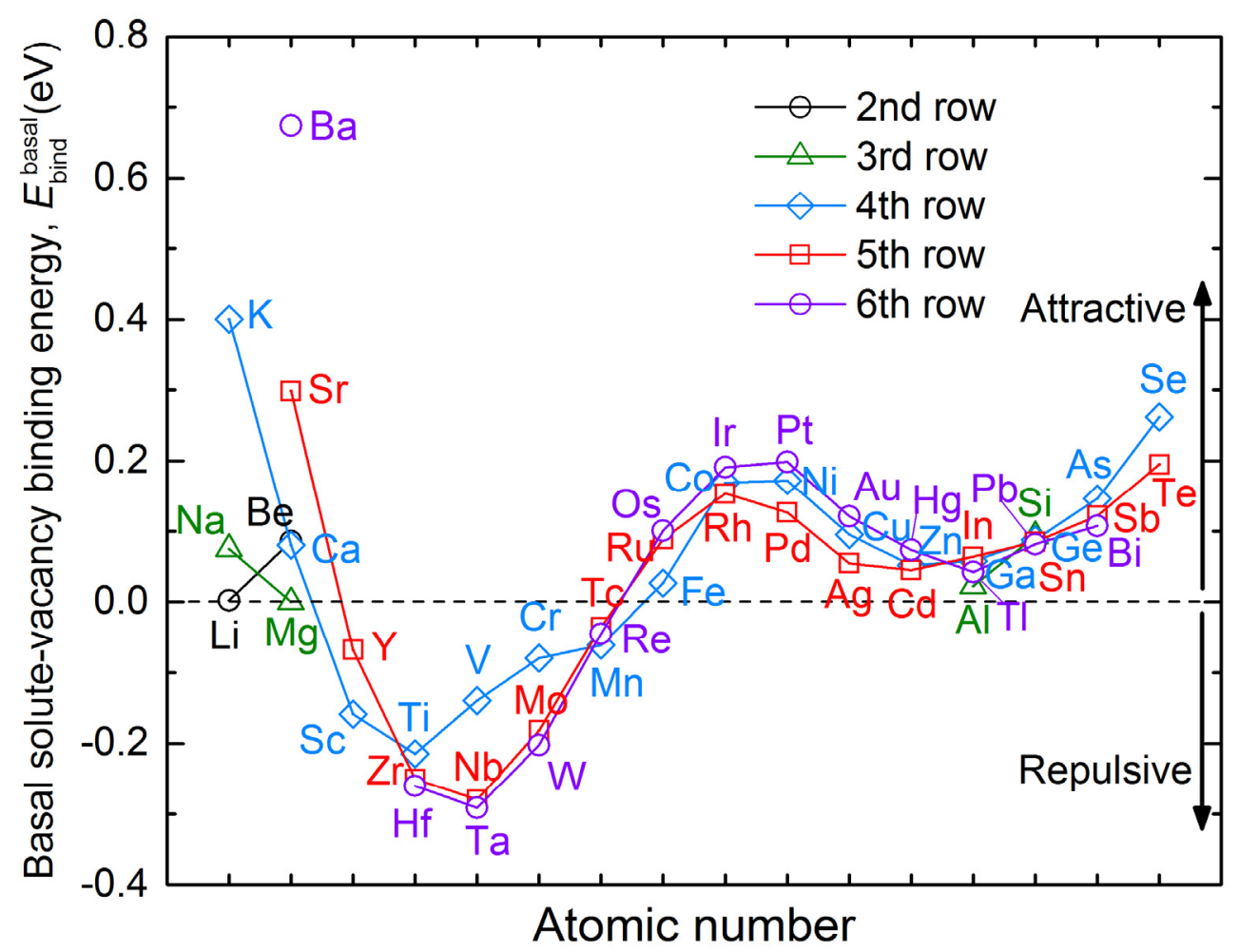

Figure 4. Calculated solute-vacancy binding energies $E_{\text {bind }}^{\text {basal }}$ of various solutes within the basal plane of hcp Mg as a function of atomic number. 


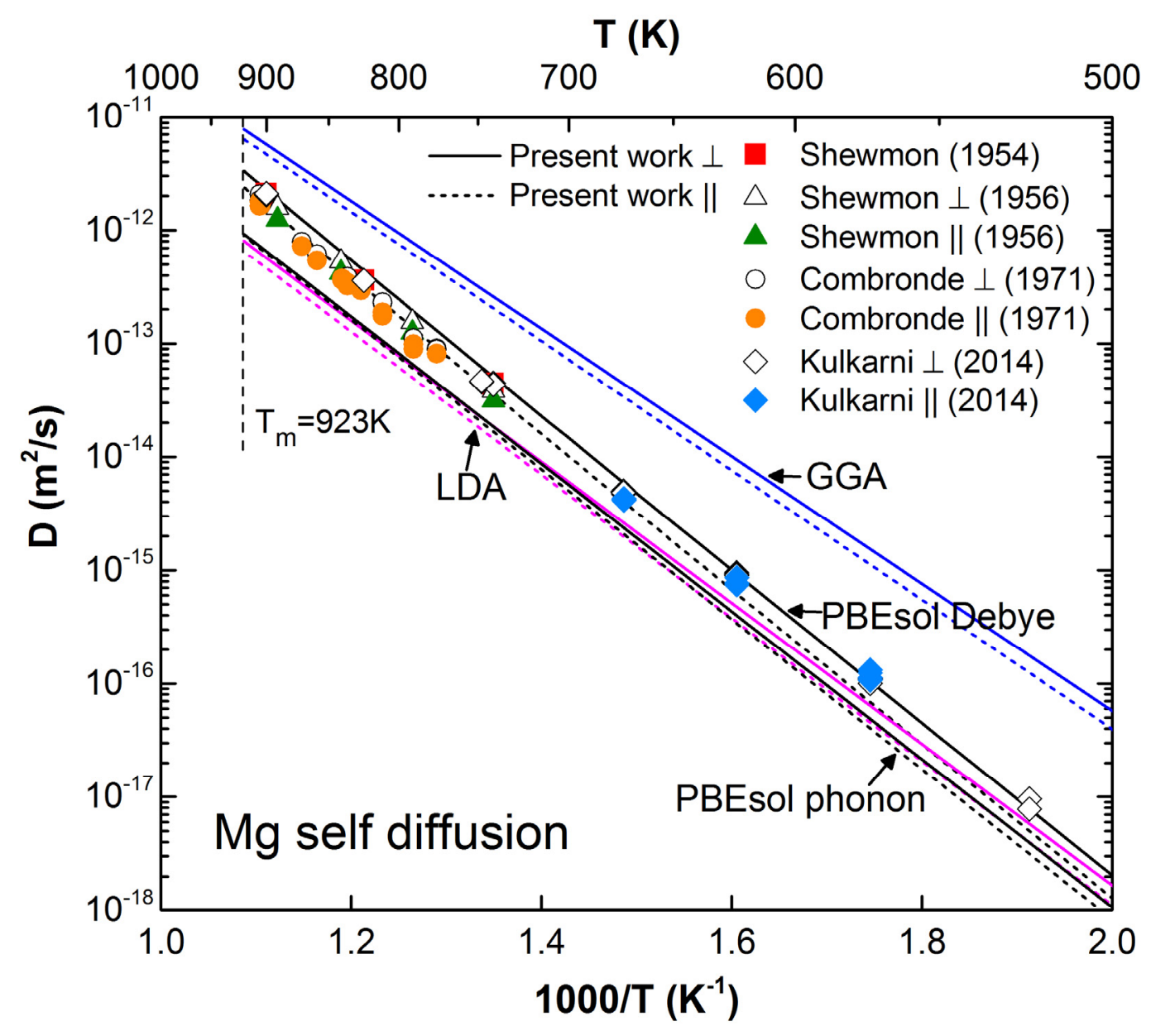

Figure 5. Predicted self-diffusion coefficients in $\mathrm{Mg}$ from different $\mathrm{X}-\mathrm{C}$ functionals of LDA, GGA, and PBEsol compared with experimental data in the literature. Calculated results with PBEsol Debye and PBEsol phonon are from the present work (black lines), results with GGA (blue lines) and LDA (pink lines) are from Ganeshan et al. [25]. Experimental data are taken from Shewmon [69, 70], Combronde and Brebec [71], and Kulkarni et al. [72]. 

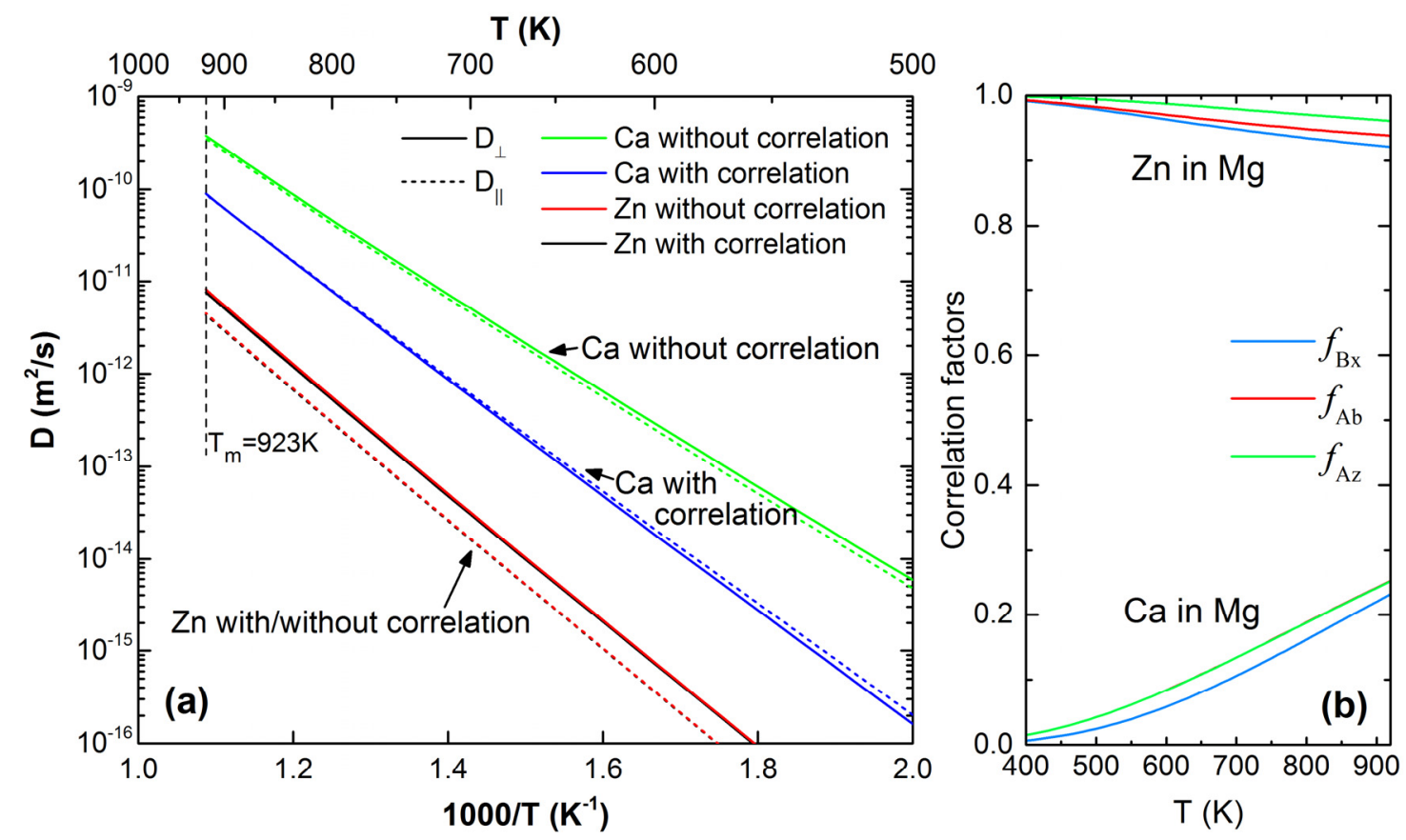

Figure 6. (a) Predicted diffusion coefficients of $\mathrm{Ca}$ and $\mathrm{Zn}$ in $\mathrm{Mg}$ with and without correlation effects considered. Note that $\mathrm{Zn}$ diffusion coefficients with and without correlation effects almost overlap with each other. (b) Calculated correlation factors $f_{B x}, f_{A b}$, and $f_{A z}$ of $\mathrm{Zn}$ and $\mathrm{Ca}$ diffusion in $\mathrm{Mg}$. Note that for the Ca correlation factors, $f_{A b}$ and $f_{A z}$ have very similar values. 


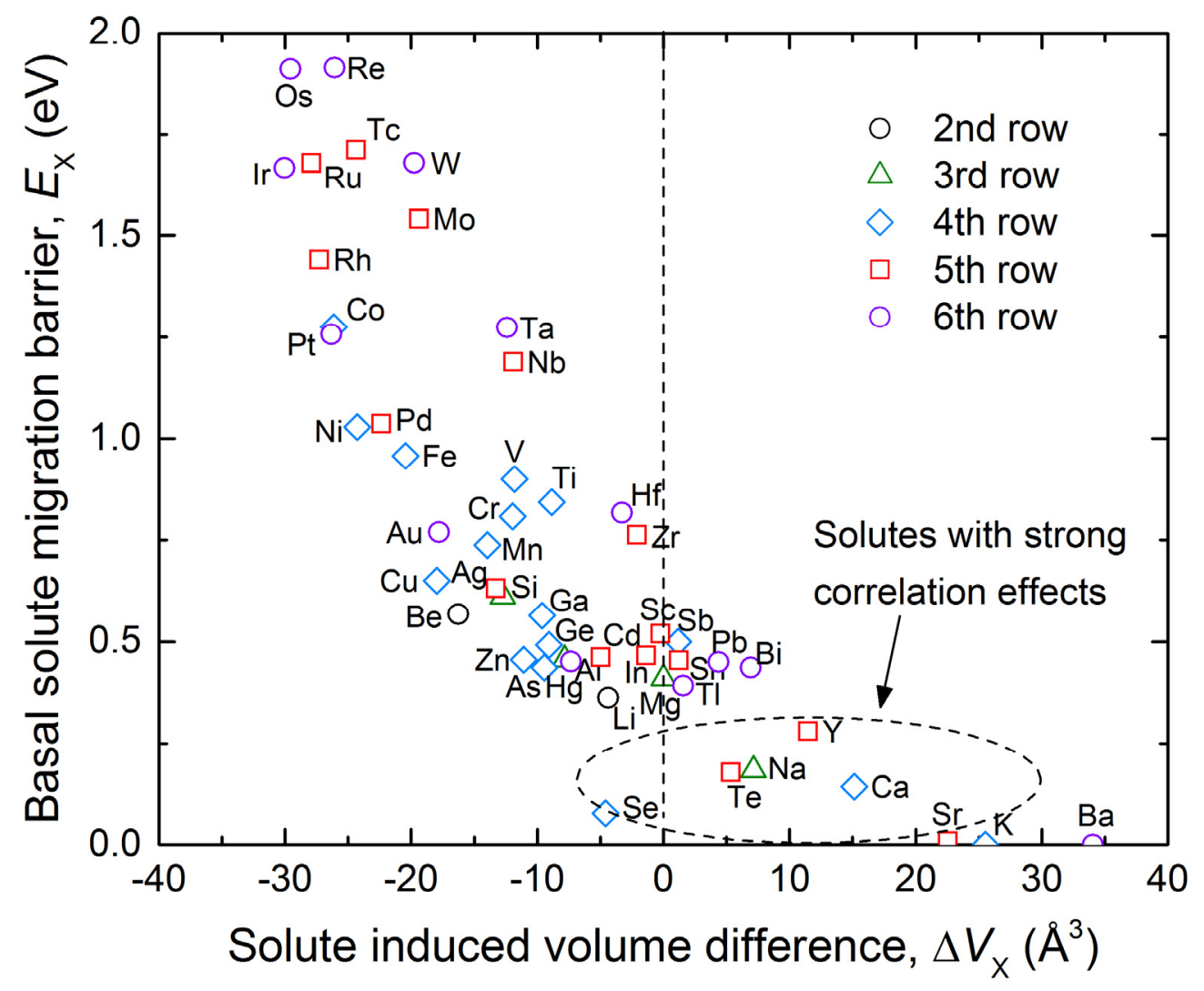

Figure 7. Calculated basal migration barrier $E_{X}$ for each solute $\mathrm{X}$ in the dilute $\mathrm{Mg}_{63} \mathrm{X}\left(\mathrm{Mg}_{95} \mathrm{X}\right.$ for $\mathrm{Ba}, \mathrm{Bi}, \mathrm{Ca}, \mathrm{K}, \mathrm{Pb}, \mathrm{Sr}$, and $\mathrm{Y}$ ) systems as a function of solute induced volume difference $\Delta V_{X}$. 

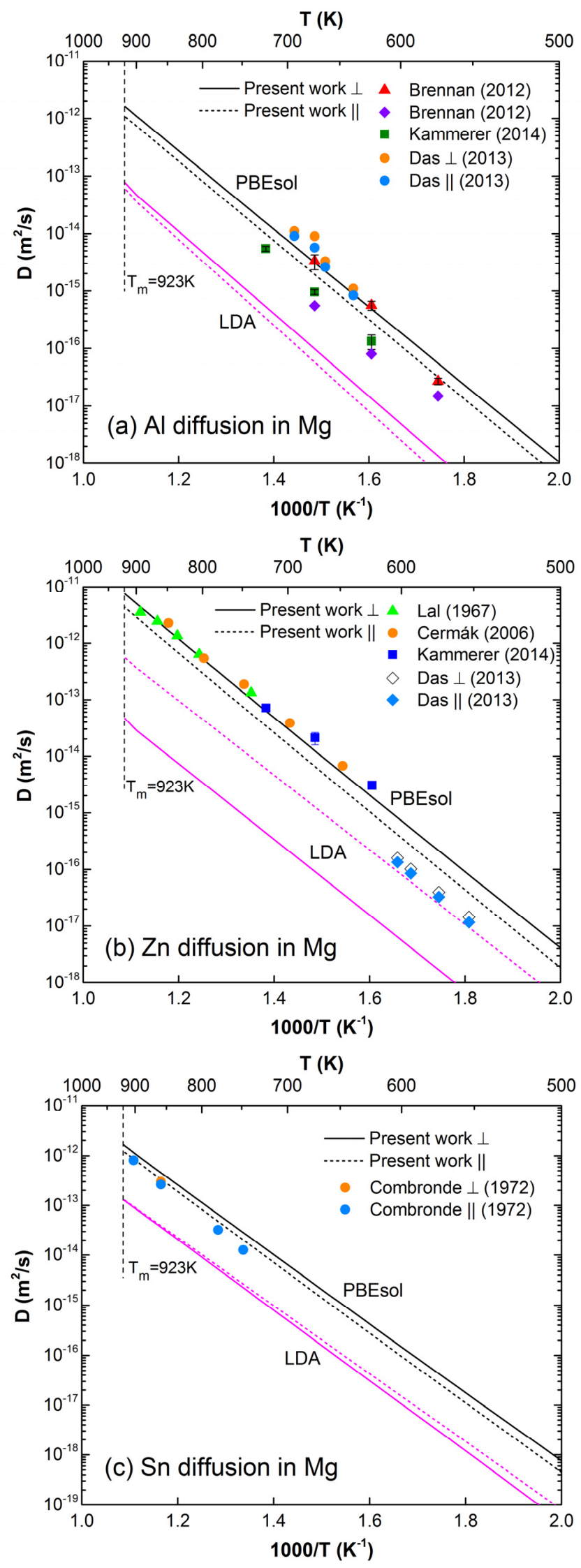
Figure 8. Predicted dilute solute tracer diffusion coefficients of (a) $\mathrm{Al}$, (b) $\mathrm{Zn}$, and (c) $\mathrm{Sn}$ in $\mathrm{Mg}$ along with available experimental data. Results with LDA are from Ganeshan et al. [16]. Al diffusion data are taken from Brennan et al. [83, 84], Kammerer et al. [88], and Das et al. [85]; Zn diffusion data are taken from Lal [77], Čermák and Stloukal [82], Kammerer et al. [88], and Das et al. [86]; Sn diffusion data are taken from Combronde and Brebec [79]. 


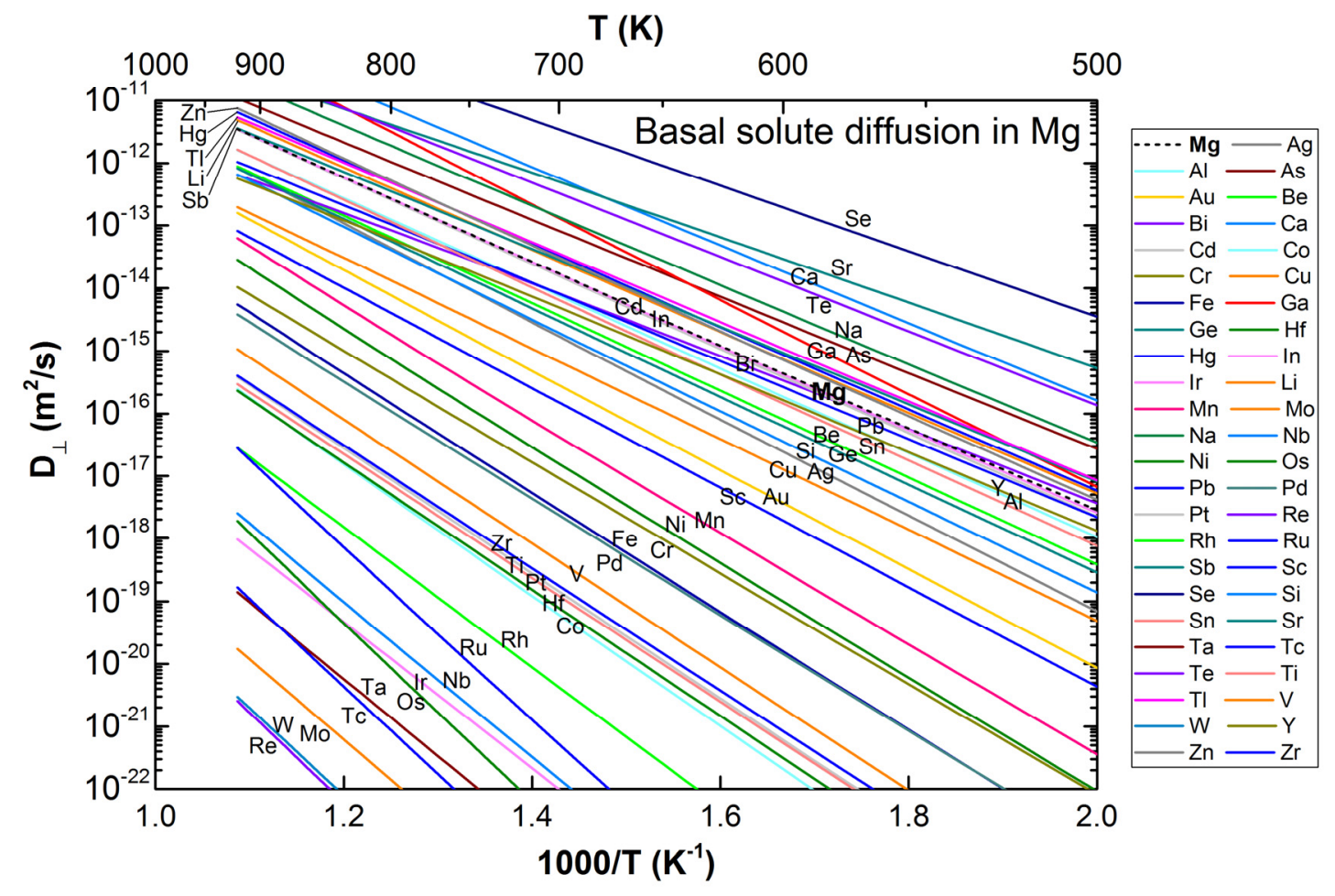

Figure 9. Predicted basal dilute solute tracer diffusion coefficients $D_{\perp}$ of 47 solutes in hcp $\mathrm{Mg}$.

The basal self-diffusion coefficient of $\mathrm{Mg}$ is plotted in a dashed line. 


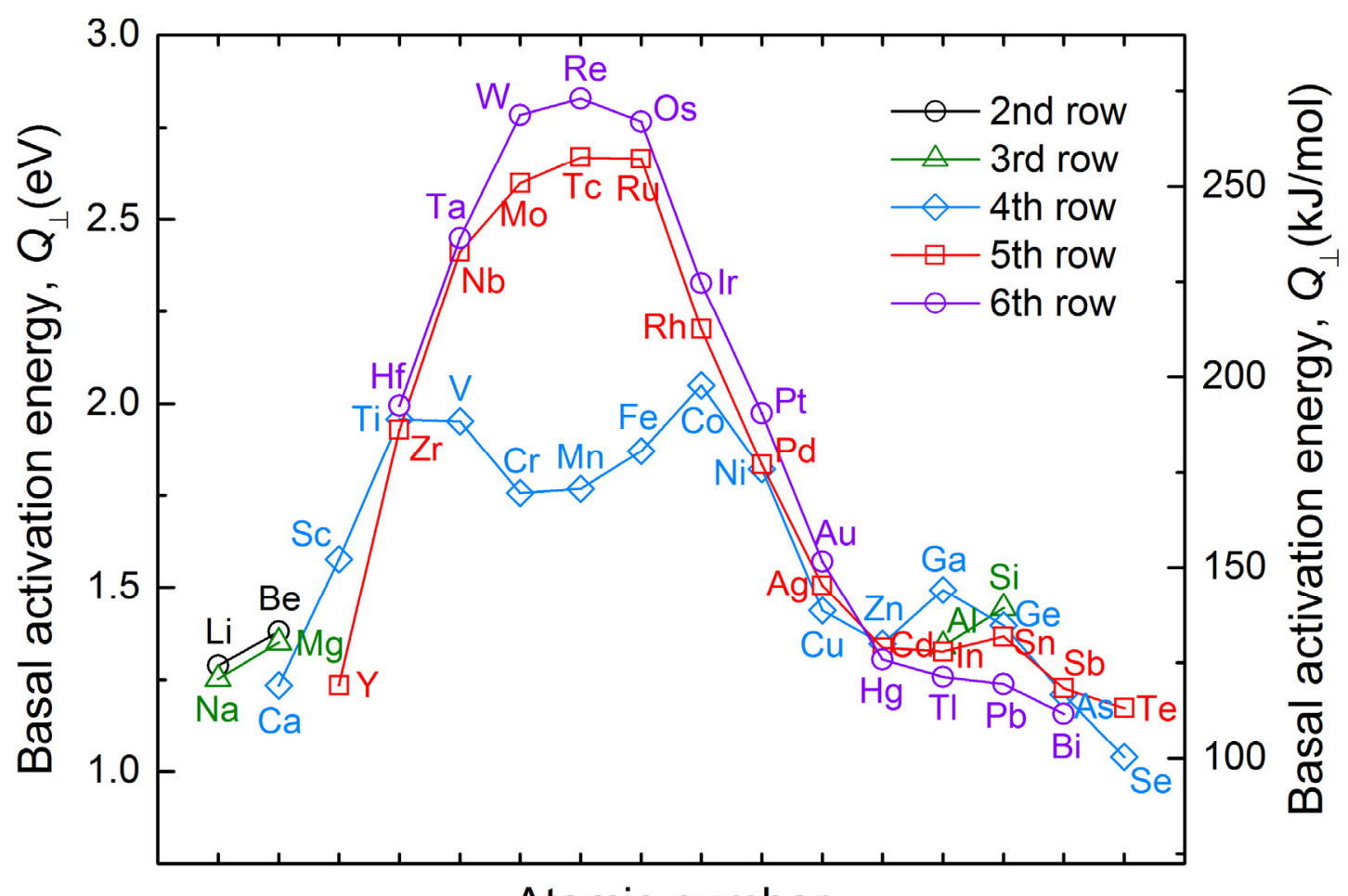

Atomic number

Figure 10. Calculated activation energies $Q_{\perp}$ of basal diffusion coefficients of various solutes in $\mathrm{Mg}$ as a function of atomic number, see Table 4 for values. 


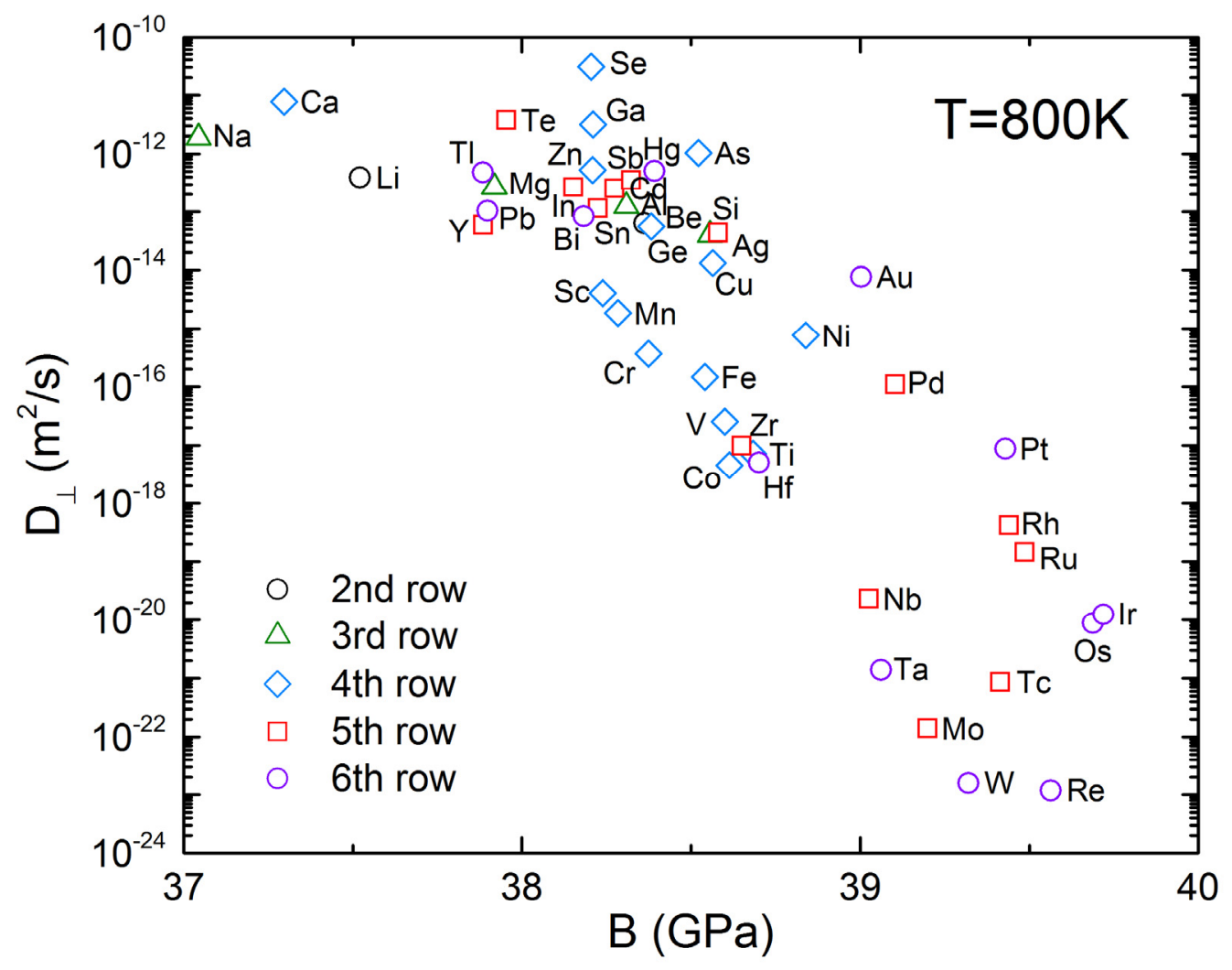

Figure 11. Basal diffusion coefficients $D_{\perp}$ at $800 \mathrm{~K}$ for each solute in the dilute $\mathrm{Mg}_{63} \mathrm{X}\left(\mathrm{Mg}_{95} \mathrm{X}\right.$ for $\mathrm{Bi}, \mathrm{Ca}, \mathrm{Pb}, \mathrm{Sr}$, and $\mathrm{Y}$ ) systems as a function of bulk modulus. 


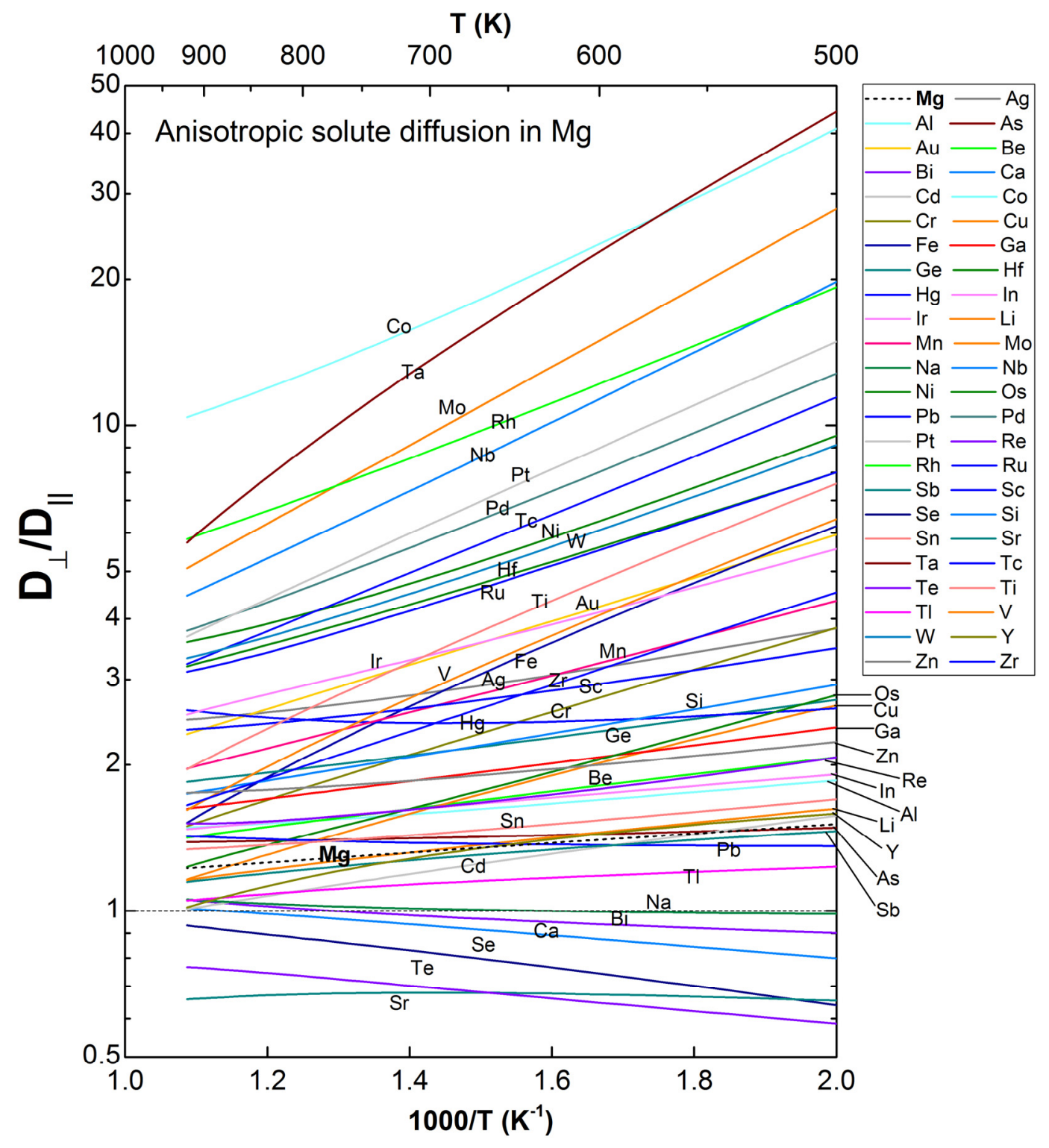

Figure 12. The ratio of predicted basal dilute solute diffusion coefficient over the non-basal one, $D_{\perp} / D_{\square}$, for 47 solutes in hcp $\mathrm{Mg}$. The ratio of self-diffusion coefficients of $\mathrm{Mg}$ is plotted in a dashed line. 


\section{Graphical Abstract}
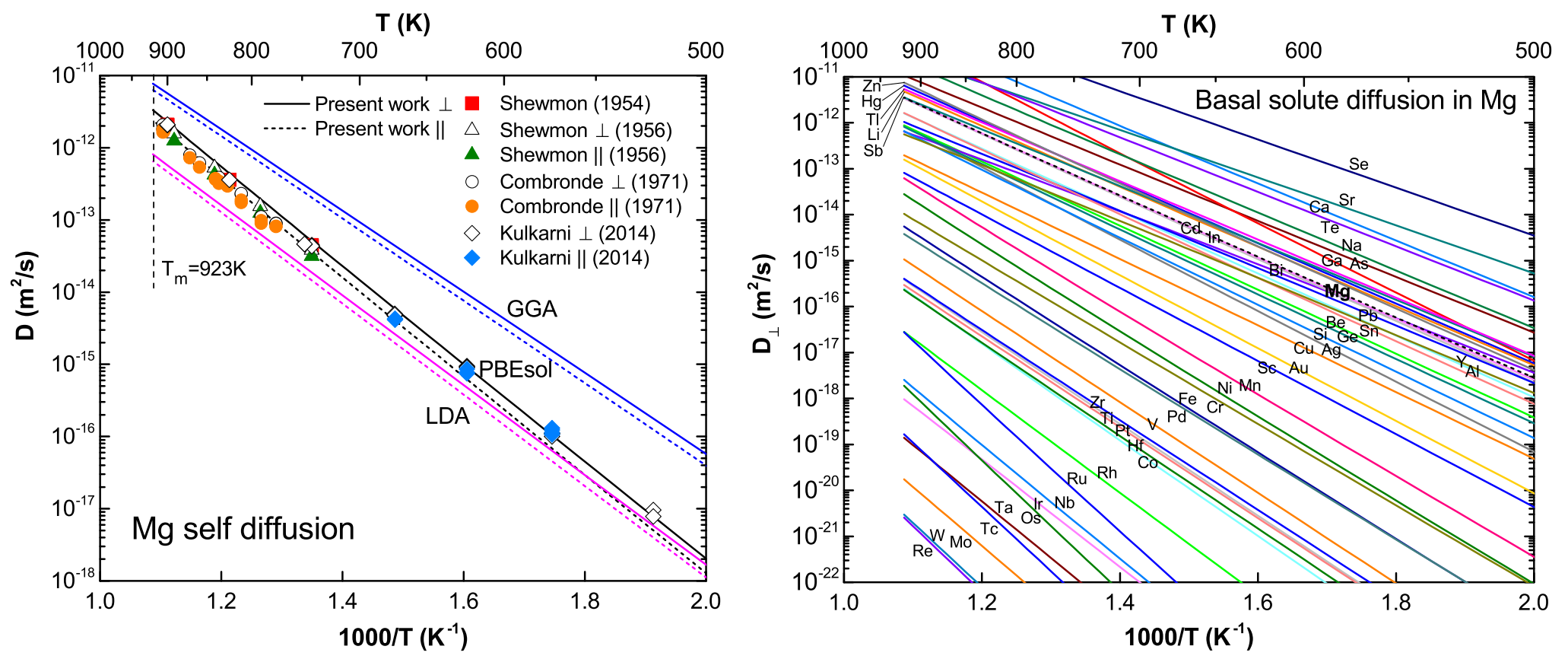